\title{
Toward an Estimation of Nadir Objective Vector Using a Hybrid of Evolutionary and Local Search Approaches
}

\author{
Kalyanmoy Deb, Kaisa Miettinen, and Shamik Chaudhuri
}

\begin{abstract}
A nadir objective vector is constructed from the worst Pareto-optimal objective values in a multiobjective optimization problem and is an important entity to compute because of its significance in estimating the range of objective values in the Pareto-optimal front and also in executing a number of interactive multiobjective optimization techniques. Along with the ideal objective vector, it is also needed for the purpose of normalizing different objectives, so as to facilitate a comparison and agglomeration of the objectives. However, the task of estimating the nadir objective vector necessitates information about the complete Pareto-optimal front and has been reported to be a difficult task, and importantly an unsolved and open research issue. In this paper, we propose certain modifications to an existing evolutionary multiobjective optimization procedure to focus its search toward the extreme objective values and combine it with a reference-point based local search approach to constitute a couple of hybrid procedures for a reliable estimation of the nadir objective vector. With up to 20-objective optimization test problems and on a three-objective engineering design optimization problem, one of the proposed procedures is found to be capable of finding the nadir objective vector reliably. The study clearly shows the significance of an evolutionary computing based search procedure in assisting to solve an age-old important task in the field of multiobjective optimization.
\end{abstract}

Index Terms-Evolutionary multiobjective optimization (EMO), hybrid procedure, ideal point, multiobjective optimization, multiple objectives, nadir point, nondominated sorting GA, Pareto optimality.

\section{INTRODUCTION}

I $\mathrm{N}$ a multiobjective optimization procedure, the estimation of a nadir objective vector (or simply a nadir point) is often an important task. The nadir objective vector is constructed from the worst values of each objective function corresponding to the entire set of Pareto-optimal solutions, that is, the Pareto-

Manuscript received July 18, 2008; revised March 21, 2009. Date of publication August 26, 2010; date of current version November 30, 2010. The work of K. Deb was supported by the Academy of Finland under Grant FiDiPro 119319 and the Foundation of Helsinki School of Economics. The work of K. Miettinen was supported in part by the Jenny and Antti Wihuri Foundation, Finland.

K. Deb is with the Department of Mechanical Engineering, Indian Institute of Technology (IIT), Kanpur 208016, India and also with the Aalto University School of Economics, Aalto FI-00076, Finland (e-mail: deb@iitk.ac.in).

K. Miettinen is with the Department of Mathematical Information Technology, University of Jyväskylä FI-40014, Jyväskylä, Finland (e-mail: kaisa.miettinen@jyu.fi).

S. Chaudhuri is with the General Electric India Technology Center, Bangalore 560066, India (e-mail: shamikc@gmail.com).

Digital Object Identifier 10.1109/TEVC.2010.2041667 optimal front. Sometimes, this point is confused with the point representing the worst objective values of the entire search space, which is often an over-estimation of the true nadir objective vector. The importance of finding the nadir objective vector was recognized by the multiple criteria decision making (MCDM) researchers and practitioners since the early 1970s. However, even after about 40 years of active research in multiobjective optimization and decision making, there does not exist a reliable procedure of finding the nadir point in problems having more than three objectives. For this reason, a reliable estimation of the nadir point is an important matter to anyone interested in multiobjective optimization, including evolutionary multiobjective optimization (EMO) researchers and practitioners. We outline here the motivation and need for finding the nadir point.

1) Along with the ideal objective vector (a point constructed from the best values of each objective), the nadir objective vector can be used to normalize objective functions [1], a matter often desired for an adequate functioning of multiobjective optimization algorithms in the presence of objective functions with different magnitudes. With these two extreme values, the objective functions can be scaled so that each scaled objective takes values more or less in the same range. These scaled values can be used for optimization with different algorithms like the reference-point method, weighting method, compromise programming, the Tchebycheff method (see [1] and references therein), or even for EMO algorithms. Such a scaling procedure may help in reducing the computational cost by solving the problem faster [2].

2) The second motivation comes from the fact that the nadir objective vector is a pre-requisite for finding preferred Pareto-optimal solutions in different interactive algorithms, such as the guess method [3] (where the idea is to maximize the minimum weighted deviation from the nadir objective vector), or it is otherwise an integral part of an interactive method like the nondifferentiable interactive multiobjective bundle-based optimization system (NIMBUS) method [1], [4]. The knowledge of a nadir point should also help in interactive EMO procedures, one implementation of which has been suggested recently [5] and many other possibilities are discussed in [6]. 
3) Thirdly, the knowledge of nadir and ideal objective values helps the decision-maker in adjusting her/his expectations on a realistic level by providing the range of each objective and can then be used to aid in specifying preference information in interactive methods in order to focus on a desired region of the Pareto-optimal front.

4) Fourthly, in visualizing a Pareto-optimal front, the knowledge of the nadir objective vector is crucial. Along with the ideal point, the nadir point provides the range of each objective in order to facilitate comparison of different Pareto-optimal solutions, that is, visualizing the trade-off information through value paths, bar charts, petal diagrams, and so on [1], [7].

5) Above all, the task of accurately estimating the nadir point in a three or more objective problems is a nontrivial and challenging task, and is an open research topic till to date. Researchers have repeatedly shown that the task is difficult even for linear multiobjective optimization problems. Therefore, any new effort to arrive at a suitable methodology for estimating the nadir point has an intellectual and pedagogic importance, despite its practical significance outlined above.

These motivations for estimating the nadir point led the researchers dealing with MCDM methodologies to suggest procedures for approximating the nadir point using a socalled payoff table [8]. This involves computing individual optimum solutions for objectives, constructing a payoff table by evaluating other objective values at these optimal solutions, and estimating the nadir point from the worst objective values from the table. This procedure may not guarantee a true estimation of the nadir point for more than two objectives. Moreover, the estimated nadir point can be either an overestimation or an under-estimation of the true nadir point. For example, Iserman and Steuer [9] have demonstrated these difficulties for finding a nadir point using the payoff table method even for linear problems and emphasized the need of using a better method. Among others, Dessouky et al. [10] suggested three heuristic methods and Korhonen et al. [11] another heuristic method for this purpose. Let us point out that all these methods suggested have been developed for linear multiobjective problems where all objectives and constraints are linear functions of the variables.

In [12], an algorithm for deriving the nadir point is proposed based on subproblems. In other words, in order to find the nadir point for an $M$-objective problem, Pareto-optimal solutions of all $\left(\begin{array}{c}M \\ 2\end{array}\right)$ bi-objective optimization problems must first be found. Such a requirement may make the algorithm computationally impractical beyond three objectives, although Szczepanski and Wierzbicki [13] implemented the above idea using evolutionary algorithms (EAs) and showed successful applications with up to four objective linear optimization problems. Moreover, the authors of [12] did not suggest how to realize the idea in nonlinear problems. It must be emphasized that because the determination of the nadir point depends on finding the worst objective values in the set of Paretooptimal solutions, even for linear problems, this is a difficult task [14].
Since an estimation of the nadir objective vector necessitates information about the whole Pareto-optimal front, any procedure of estimating this point should ideally involve finding Pareto-optimal solutions. This makes the task more difficult compared to finding the ideal point [11]. Since EMO algorithms can be used to find a representation of the entire or a part of the Pareto-optimal front, EMO methodologies stand as viable candidates for this task. Another motivation for using an EMO procedure is that nadir point estimation is to be made only once in a problem at the beginning of the decision making process before any human decision maker is involved. So, even if the proposed procedure uses somewhat substantial computational effort (one of the criticisms made often against evolutionary optimization methods), a reliable and accurate methodology for estimating the nadir point is desired in practice.

A careful thought will reveal that an estimation of the nadir objective vector may not need finding the complete Paretooptimal front, but only an adequate number of critical Paretooptimal solutions may be enough for this task. Based on this concept, an earlier preliminary study by the authors [15] showed that by altering the usual definition of a crowding distance metric of an existing EMO methodology (elitist nondominated sorting GA or NSGA-II [16]) to emphasize objective-wise best and worst Pareto-optimal solutions (we call these here extreme solutions), a near nadir point can be estimated on a number of test problems. Since this paper, we realized that the proposed NSGA-II procedure alone was not enough to find the desired extreme solutions in a finite amount of computational effort, when applied to other more tricky optimization problems. In this paper, we hybridize the previously proposed NSGA-II approach with a local search procedure which uses the idea of an achievement scalarizing function utilized, for example, in an interactive MCDM approach - the reference-point approach [17]—to enhance the convergence of solutions to the desired extreme points. This extension, by far, is not an easy task, as a local search in any form in the context of multiple conflicting objectives is a difficult proposition. Empirical results of this hybrid nadir point estimation procedure on problems with up to 20 objectives, on some difficult numerical optimization problems, and on an engineering design problem amply demonstrate the usefulness and promise of the proposed hybrid procedure.

The rest of this paper is organized as follows. In Section II, we introduce basic concepts of multiobjective optimization and discuss the importance and difficulties of estimating the nadir point. In Section III, we describe two modified NSGA-II approaches for finding near extreme Pareto-optimal solutions. The nadir point estimation procedures proposed based on a hybrid evolutionary-cum-local-search concept are described in Section IV. The performances of the modified NSGA-II procedures are tested and compared with a naive approach on a number of scalable numerical test problems and the results are described in Section V. The use of the hybrid nadir point estimation procedure in full is demonstrated in Section VI by solving three test problems, including an engineering design problem. Some discussions and possible extensions of the paper are presented in Section VII. Finally, the paper is concluded in Section VIII. 


\section{NADIR OBJeCtive Vector AND Difficulties of ITS ESTIMATION}

We consider multiobjective optimization problems involving $M$ conflicting objectives $\left(f_{i}: \mathcal{S} \rightarrow \mathbf{R}\right)$ as functions of decision variables $\mathbf{x}$

$$
\begin{aligned}
\operatorname{minimize} & \left\{f_{1}(\mathbf{x}), f_{2}(\mathbf{x}), \ldots, f_{M}(\mathbf{x})\right\} \\
\text { subject to } & \mathbf{x} \in \mathcal{S}
\end{aligned}
$$

where $\mathcal{S} \subset \mathbf{R}^{n}$ denotes the set of feasible solutions. A vector consisting of objective function values calculated at some point $\mathbf{x} \in \mathcal{S}$ is called an objective vector $\mathbf{f}(\mathbf{x})=\left(f_{1}(\mathbf{x}), \ldots, f_{M}(\mathbf{x})\right)^{T}$. Problem (1) gives rise to a set of Pareto-optimal solutions or a Pareto-optimal front $\left(P^{*}\right)$, providing a trade-off among the objectives. The domination between two solutions is defined as follows [1], [18]:

Definition 1: A solution $\mathbf{x}^{(1)}$ is said to dominate the other solution $\mathbf{x}^{(2)}$, if (i) the solution $\mathbf{x}^{(1)}$ is no worse than $\mathbf{x}^{(2)}$ in all objectives (that is, in the case of a minimization problem, $f_{i}\left(\mathbf{x}^{(1)}\right) \leq f_{i}\left(\mathbf{x}^{(2)}\right)$ for all $\left.i=1,2, \ldots, M\right)$ and (ii) the solution $\mathbf{x}^{(1)}$ is strictly better than $\mathbf{x}^{(2)}$ in at least one objective (that is, in the case of a minimization problem, $f_{i}\left(\mathbf{x}^{(1)}\right)<f_{i}\left(\mathbf{x}^{(2)}\right)$ for at least one index $i$ ).

Pareto-optimal solutions can then be defined as follows [1]:

Definition 2: A decision vector $\mathbf{x}^{*} \in \mathcal{S}$ and the corresponding objective vector $\mathbf{f}\left(\mathbf{x}^{*}\right)$ are Pareto-optimal if there does not exist another decision vector $\mathbf{x} \in S$ that dominates $\mathbf{x}^{*}$ according to Definition 1.

Let us mention that if an objective $f_{j}$ is to be maximized, it is equivalent to minimize $-f_{j}$. In what follows, we assume that the Pareto-optimal front is bounded. We now define a critical point, as follows:

Definition 3: A point $\mathbf{z}^{(j)^{c}}$ is a critical point with respect to the $j$ th objective function, if it corresponds to the worst value of $f_{j}$ among all Pareto-optimal solutions, i.e., $\mathbf{z}^{(j)^{c}}=\{\mathbf{f}(y) \mid y=$ $\left.\operatorname{argmax}_{\mathbf{X} \in P^{*}} f_{j}(\mathbf{x})\right\}$.

The nadir objective vector can now be defined as follows:

Definition 4: An objective vector $\mathbf{z}^{\text {nad }}=\left(z_{1}^{\text {nad }}, \ldots, z_{M}^{\text {nad }}\right)^{T}$ whose $j$ th element is taken from the $j$ th component of the corresponding critical Pareto-optimal point $z_{j}^{\text {nad }}=z_{j}^{(j)^{c}}$ is called a nadir objective vector.

Due to the requirement that a critical point must be a Pareto-optimal point, the estimation of the nadir objective vector is, in general, a difficult task. Unlike the ideal $o b$ jective vector $\mathbf{z}^{*}=\left(z_{1}^{*}, \ldots, z_{M}^{*}\right)^{T}$, which can be found by minimizing each objective individually over the feasible set $S$ (i.e., $z_{j}^{*}=\min _{\mathbf{x} \in \mathcal{S}} f_{j}(\mathbf{x})$ ), the nadir point cannot be formed by maximizing objectives individually over $\mathcal{S}$. To find the nadir point, Pareto-optimality of solutions used for constructing the nadir point must first be established. This makes the task of finding the nadir point a difficult one.

To illustrate this aspect, let us consider a bi-objective minimization problem shown in Fig. 1 . If we maximize $f_{1}$ and $f_{2}$ individually, we obtain points $\mathrm{A}$ and $\mathrm{B}$, respectively. These two points can be used to construct the so-called worst objective vector, $\mathbf{z}^{w}$. In many problems (even in bi-objective optimization problems), the nadir objective vector and the worst objective vector are not the same point, which can also be seen in Fig. 1.

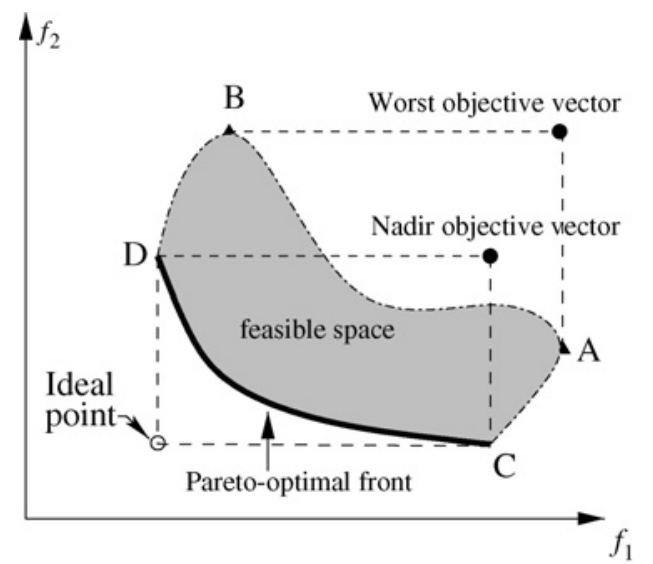

Fig. 1. Nadir and worst objective vectors may be different.

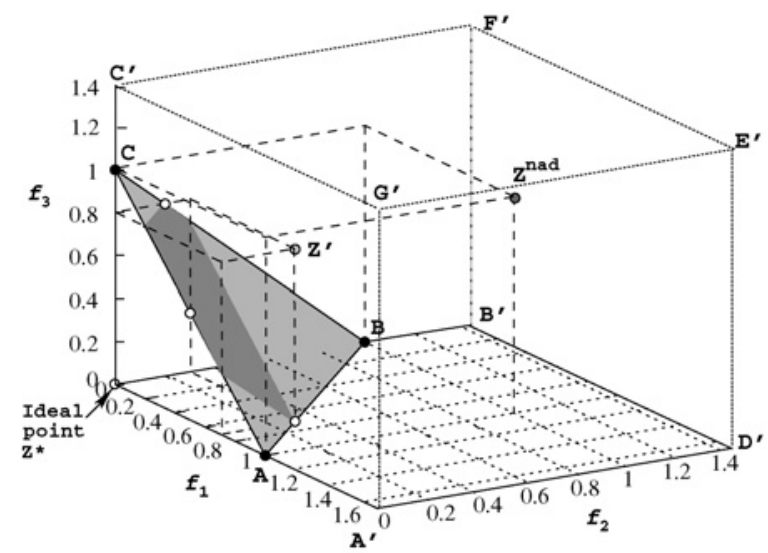

Fig. 2. Payoff table may not produce the true nadir point.

In order to estimate the nadir point correctly, we need to find critical points (such as C and D in Fig. 1).

\section{A. Payoff Table Method}

Benayoun et al. [8] introduced the first interactive multiobjective optimization method and used a nadir point (although the authors did not use the term "nadir"), which was to be found by using a payoff table. To be more specific, each objective function is first minimized individually and then a table is constructed where the $i$ th row of the table represents values of all objective functions calculated at the point where the $i$ th objective obtained its minimum value. Thereafter, the maximum value of the $j$ th column can be considered as an estimate of the upper bound of the $j$ th objective in the Paretooptimal front and these maximum values may be used as components of an approximation of the nadir objective vector. The main difficulty of such an approach is that solutions are not necessarily unique and thus corresponding to the minimum solution of an objective there may exist more than one solution having different values of other objectives, in problems having more than two objectives. In these problems, the payoff table method may not result in an accurate estimation of the nadir objective vector.

Let us consider the Pareto-optimal front of a hypothetical problem involving three objective functions shown in Fig. 2. 
The problem has a bounded objective space lying inside the rectangular outer box marked with solid lines. From the box, the region below the triangular surface $\mathrm{ABC}$ is removed to construct the feasible objective space. Since all three objectives are minimized, the Pareto-optimal front is the triangular plane $\mathrm{ABC}$. The minimum value of the first objective function is zero. As can be seen from the figure, there exist a number of solutions having a value zero for function $f_{1}$ and different combinations of $f_{2}$ and $f_{3}$ values. These solutions lie on the $f_{1}=0$ plane, but on the trapezoid $\mathrm{CBB}^{\prime} \mathrm{F}^{\prime} \mathrm{C}^{\prime} \mathrm{C}$. In the payoff table, when three objectives are minimized one at a time, we may get objective vectors $\mathbf{f}^{(1)}=(0,0,1)^{T}$ (point $\mathrm{C}), \mathbf{f}^{(2)}=(1,0,0)^{T}$ (point A), and $\mathbf{f}^{(3)}=(0,1,0)^{T}$ (point B) corresponding to minimizations of $f_{1}, f_{2}$, and $f_{3}$, respectively, and then the true nadir point $z^{\text {nad }}=(1,1,1)^{T}$ can be found. However, if vectors $\mathbf{f}^{(1)}=(0,0.2,0.8)^{T}, \mathbf{f}^{(2)}=(0.5,0,0.5)^{T}$, and $\mathbf{f}^{(3)}=(0.7,0.3,0)^{T}$ (marked with open circles) are found as minimum points from corresponding minimizations of $f_{1}$, $f_{2}$, and $f_{3}$, respectively, a wrong estimate $\mathbf{z}^{\prime}=(0.7,0.3,0.8)^{T}$ of the nadir point will be made. The figure shows how such a wrong nadir point represents only a portion (shown darkshaded) of the Pareto-optimal front. Here we obtained an underestimation but the result may also be an overestimation of the true nadir point in some other problems. Thus, we need a more reliable method to estimate the nadir point.

\section{EVOlutionARY MULTIOBJECTIVE APPROACHES FOR NADIR POINT ESTIMATION}

As has been discussed so far, the nadir point is associated with Pareto-optimal solutions and, thus, determining a set of Pareto-optimal solutions will facilitate the estimation of the nadir point. For the past decade or so, EMO algorithms have been gaining popularity because of their ability to find multiple, wide-spread, Pareto-optimal solutions simultaneously [18], [19]. Since they aim at finding a set of Pareto-optimal solutions, an EMO approach may be an ideal way to find multiple critical points simultaneously for an estimation of the nadir objective vector. Let us now discuss several existing approaches for estimating the nadir point using an EMO approach.

\section{A. Naive Approach}

In the so-called naive approach, first a well-distributed set of Pareto-optimal solutions can be attempted to be found by an EMO, as was also suggested in [15]. Thereafter, an estimate of the nadir objective vector can be made by picking the worst values of each objective. This idea was implemented in [13] and applied to a couple of three and four objective optimization problems. However, this naive approach of first finding a representative set of Pareto-optimal solutions and then determining the nadir objective vector seems to possess some difficulties. In the context of the problem depicted in Fig. 2, this means first finding a well-represented set of solutions on the plane $\mathrm{ABC}$ and then estimating the nadir point from them.

Recall that one of the main purposes of estimating the nadir objective vector is that along with the ideal point, it can be used to normalize different objective functions, so that an interactive multiobjective optimization algorithm can be used to find the most preferred Pareto-optimal solution. But by the naive approach, an EMO is already utilized to find a representative set of Pareto-optimal solutions. One may think that there is no apparent reason for constructing the nadir point for any further analysis.

However, representing and analyzing the set of Paretooptimal solutions is not trivial when we have more than two objectives in question. Furthermore, we can list several other difficulties related to the above-described simple approach. Recent studies have shown that EMO approaches using the domination principle possess a number of difficulties in solving problems having a large number of objectives [20]-[22].

1) To properly represent a high-dimensional Pareto-optimal front requires an exponentially large number of points [18], thereby requiring a large computational cost.

2) With a large number of conflicting objectives, a large proportion of points in a random initial population are nondominated to each other. Since EMO algorithms emphasize all nondominated solutions in a generation, a large portion of an EA population gets copied to the next generation, thereby allowing only a small number of new solutions to be included in a generation. This severely slows down the convergence of an EMO toward the true Pareto-optimal front.

3) EMO methodologies maintain a good diversity of nondominated solutions by explicitly using a nichepreserving scheme which uses a diversity metric specifying how diverse the nondominated solutions are. In a problem with many objectives, defining a computationally fast yet a good indicator of higher-dimensional distances among solutions becomes a difficult task. This aspect also makes the EMO approaches computationally expensive.

4) With a large number of objectives, visualization of a large-dimensional Pareto-optimal front gets difficult.

The above-mentioned shortcomings cause EMO approaches to be inadequate for finding the complete Pareto-optimal front in the first place [21]. Thus, for handling a large number of objectives, it may not be advantageous to use the naive approach in which an EMO is employed to first find a representative set of points on the entire Pareto-optimal front and then construct the nadir point from these points.

\section{B. Multiple Bi-Objective Formulations}

Szczepanski and Wierzbicki [13] have simulated the idea of solving multiple bi-objective optimization problems suggested in [12] using an EMO approach and constructing the nadir point by accumulating all bi-objective Pareto-optimal solutions together. In the context of the three-objective optimization problem described in Fig. 2 for which the Pareto-optimal front is the plane $\mathrm{ABC}$, minimization of the pair $f_{1}-f_{2}$ will correspond to one Pareto-optimal objective vector having a value of zero for both objectives. An easy way to visualize the objective space for the $f_{1}-f_{2}$ optimization problem is to project every point from the above $3-\mathrm{D}$ objective space on 
the $f_{1}-f_{2}$ plane. The projected objective space lies on the first quadrant of the $f_{1}-f_{2}$ plane and the origin [the point $(0,0)$ corresponding to $\left.\left(f_{1}, f_{2}\right)\right]$ is the only Pareto-optimal point to the above problem. However, this optimal objective vector $\left(f_{1}=0\right.$ and $\left.f_{2}=0\right)$ corresponds to any value of the third objective function lying on the line $\mathrm{CC}^{\prime}$ (since the third objective was not considered in the above bi-objective optimization process). The authors of [13] have also suggested the use of an objective-space niching technique to find a set of well-spread optimal solutions on the objective space. But since all objective vectors on the line $\mathrm{CC}^{\prime}$ correspond to an identical $\left(f_{1}, f_{2}\right)$ value of $(0,0)$, the objective-space niching will not have any motivation to find multiple solutions on the line $\mathrm{CC}^{\prime}$. Thus, to find multiple solutions on the line $\mathrm{CC}^{\prime}$ so that the point $\mathrm{C}$ can be captured by this bi-objective optimization task to make a correct estimate of the nadir point, an additional variable-space niching [23], [24] must also be used to get a well-spread set of solutions on the line $\mathrm{CC}^{\prime}$. This aspect was ignored in [13], but it is important to note that in order to accurately estimate the nadir point, any arbitrary objective vector on the line $\mathrm{CC}^{\prime}$ will not be adequate, but the point $\mathrm{C}$ must be accurately found. Similarly, the other two pair-wise minimizations, if performed with a variable-space niching, will give rise to sets of solutions on the lines $\mathrm{AA}^{\prime}$ and $\mathrm{BB}^{\prime}$. According to the procedure of [13], all these points (objective vectors) can then be put together, dominated solutions can be eliminated, and the nadir point can be estimated from the remaining nondominated points. If only exact objective vectors $\mathrm{A}, \mathrm{B}$, and $\mathrm{C}$ are found by respective pair-wise minimizations, the above procedure will result in finding critical points A, B, and $\mathrm{C}$, thereby making a correct estimate of the nadir point $\left(\mathbf{z}^{\mathrm{nad}}\right)$.

Although the idea seems interesting and theoretically sound, it requires $\left(\begin{array}{c}M \\ 2\end{array}\right)$ bi-objective optimizations with both objective and variable-space niching methodologies to be performed. This may be a daunting task particularly for problems having more than three or four objectives. Moreover, the outcome of the procedure will depend on the chosen niching parameter on both objective and decision-space niching operators.

However, the idea of concentrating on a preferred region on the Pareto-optimal front, instead of finding the entire Pareto-optimal front, can be pushed further. Instead of finding bi-objective Pareto-optimal fronts by several pair-wise optimizations, an emphasis can be placed in an EMO approach to find only the critical points of the Pareto-optimal front. These points are nondominated points which will be required to estimate the nadir point correctly. With this change in focus, an EMO approach can also be used to handle largedimensional problems, particularly since the focus would be to only converge to the extreme points on the Pareto-optimal front, instead of aiming at maintaining diversity. For the three-objective minimization problem of Fig. 2, the proposed EMO approach would then distribute its population members near the extreme points $\mathrm{A}, \mathrm{B}$, and $\mathrm{C}$ (instead of the entire Pareto-optimal front $\mathrm{ABC}$ or nonoptimal solutions), so that the nadir point can be estimated quickly. Our earlier paper [15] suggested the following two approaches.

\section{Worst-Crowded NSGA-II Approach}

We discuss this approach for an implementation on a particular EMO approach (NSGA-II [16]), but the concept can, in principle, be implemented on other state-of-the-art EMO approaches as well. Since the nadir point must be constructed from the worst objective values of Pareto-optimal solutions, it is intuitive to think of an idea in which population members having the worst objective values within a nondominated front are emphasized. For this, we suggested a modified crowding distance scheme in NSGA-II by emphasizing the worst objective values in every nondominated front [15]. We called this by the name "Worst-Crowded NSGA-II Approach."

In every generation, population members on every nondominated front (having $N_{f}$ members) are first sorted from their minimum to maximum values based on each objective (for minimization problems) and a rank equal to the position of the solution in the sorted list is assigned. In this way, a member $i$ in a front gets a rank $R_{i}^{(m)}$ from the sorting in the $m$ th objective. The solution with the minimum function value in the $m$ th objective gets a rank value $R_{i}^{(m)}=1$ and the solution with the maximum function value in the $m$ th objective gets a rank value $R_{i}^{(m)}=N_{f}$. Such a rank assignment continues for all $M$ objectives. Thus, at the end of this assignment process, each solution in the front gets $M$ ranks, one corresponding to each objective function. Thereafter, the crowding distance $d_{i}$ to a solution $i$ in the front is assigned as the maximum of all $M$ ranks

$$
d_{i}=\max \left\{R_{i}^{(1)}, R_{i}^{(2)}, \ldots, R_{i}^{(M)}\right\} .
$$

In this way, the solution with the maximum objective value of any objective gets the highest crowding distance. Thus, the NSGA-II approach emphasizes a solution if it lies on a better nondominated front and also if it has a higher crowding distance value for solutions of the same nondominated front. This dual task of selecting nondominated solutions and solutions with worst objective values should, in principle, lead to a proper estimation of the nadir point.

However, we realize that an emphasis on the worst nondominated points alone may have at least two difficulties in certain problems. First, since the focus is to find only a few solutions (instead of a complete front), the population may lose its diversity early on during the search process, thereby slowing down the progress toward the critical points. Moreover, if, for some reason, the convergence is a premature event to wrong solutions, the lack of diversity among population members will make it even harder for the EMO algorithm to recover and find the necessary critical solutions to construct the true nadir point.

The second difficulty of the worst-crowded NSGA-II approach may occur in certain problems, in which an identification of critical points alone from the Pareto-optimal front is not enough. Some spurious non-Pareto-optimal points can remain nondominated with the critical points in a population and may make a wrong estimate of the nadir point. Let us discuss this important issue with an example problem. Consider a threeobjective minimization problem shown in Fig. 3, where the surface $\mathrm{ABCD}$ represents the Pareto-optimal front. 


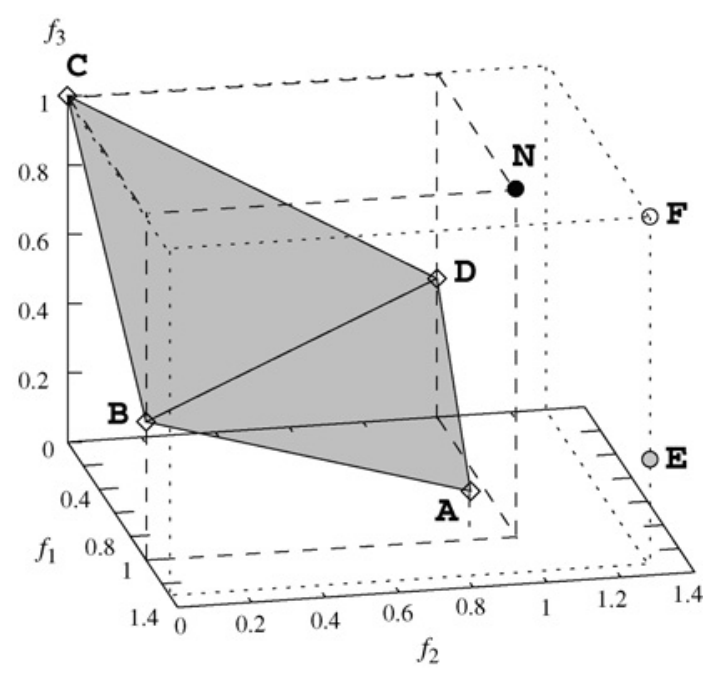

Fig. 3. Problem which may cause difficulty to the worst-crowded approach.

The true nadir point is at $\mathbf{z}^{\text {nad }}=(1,1,1)^{T}$. By using the worst-crowded NSGA-II, we expect to find three individual critical points: $\mathrm{B}=(1,0,0.4)^{T}$ (for $\left.f_{1}\right), \mathrm{D}=(0,1,0.4)^{T}$ (for $\left.f_{2}\right)$, and $\mathrm{C}=(0,0,1)^{T}$ (for $\left.f_{3}\right)$. Note that there is no motivation for the worst-crowded NSGA-II to find and maintain point $\mathrm{A}=(0.9,0.9,0.1)^{T}$ in the population, as this point does not correspond to the worst value of any of the three objectives in the set of Pareto-optimal solutions. With the three points (B, C, and $\mathrm{D}$ ) in a population, a non-Pareto-optimal point $E$ [with an objective vector $\left.(1.3,1.3,0.3)^{T}\right]$, if found by genetic operators, will become nondominated to points $\mathrm{B}, \mathrm{C}$, and $\mathrm{D}$, and will continue to exist in the population. Thereafter, the worst-crowded NSGA-II will emphasize points $\mathrm{C}$ and $\mathrm{E}$ as extreme points and the reconstructed nadir point will become $\mathrm{F}=(1.3,1.3,1.0)^{T}$, which is a wrong estimation. This difficulty could have been avoided, if the point $\mathrm{A}$ was somehow present in the population.

A little thought will reveal that the point $\mathrm{A}$ is a Paretooptimal solution, but corresponds to the best value of $f_{3}$. If the point $\mathrm{A}$ is present in the population, it will dominate points like $\mathrm{E}$ and would not allow points like $\mathrm{E}$ to be present in the nondominated front. Interestingly, this situation does not occur in bi-objective optimization problems. To avoid a wrong estimation of the nadir point due to the above difficulty, ideally, an emphasis on maintaining all Pareto-optimal solutions in the population must be made. But, since this is not practically viable for a large number of objectives (as discussed in Section III-A), we discuss another approach which deals with the above-mentioned difficulties better than the worst-crowded approach.

\section{Extremized-Crowded NSGA-II Approach}

In the extremized-crowded NSGA-II approach, in addition to emphasizing the worst solution corresponding to each objective, we also emphasized the best solution corresponding to every objective [15]. We refer to the individual best and worst Pareto-optimal solutions as "extreme" solutions here. In the extremized-crowded NSGA-II approach, solutions on a particular nondominated front are first sorted from minimum

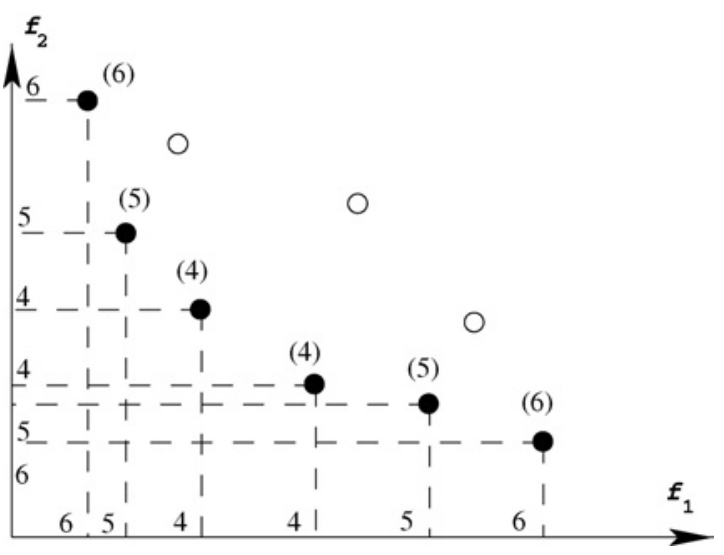

Fig. 4. Crowding distance computation procedure in extremized-crowded NSGA-II approach.

(with rank $R_{i}^{(m)}=1$ ) to maximum (with rank $=N_{f}$ ) based on each objective. A solution closer to either extreme objective values (minimum or maximum objective values) gets a higher rank compared to that of an intermediate solution. Thus, the rank of solution $i$ for the $m$ th objective $R_{i}^{(m)}$ is reassigned as $\max \left\{R_{i}^{(m)}, N_{f}-R_{i}^{(m)}+1\right\}$. Two extreme solutions for every objective get a rank equal to $N_{f}$ (number of solutions in the nondominated front), the solutions next to these extreme solutions get a rank $\left(N_{f}-1\right)$, and so on. Fig. 4 shows this rankassignment procedure. After a rank is assigned to a solution by each objective, the maximum value of the assigned ranks is declared as the crowding distance, as in (2). The final crowding distance values are shown within brackets in Fig. 4.

For a problem having a 1-D Pareto-optimal front (such as, in a bi-objective problem), the above crowding distance assignment is similar to the worst crowding distance assignment scheme (as the minimum-rank solution of one objective is also the maximum-rank solution of at least one other objective). However, for problems having a higher-dimensional Paretooptimal hyper-surface, the effect of extremized crowding is different from that of the worst-crowded approach. In the three-objective problem shown in Fig. 3, the extremizedcrowded approach will not only emphasize the extreme points $\mathrm{A}, \mathrm{B}, \mathrm{C}$, and $\mathrm{D}$, but also solutions on edges $\mathrm{CD}$ and $\mathrm{BC}$ (having the smallest $f_{1}$ and $f_{2}$ values, respectively) and solutions near them. This approach has two advantages: 1) a diversity of solutions in the population will be maintained thereby allowing genetic operators (recombination and mutation) to find better solutions and not cause a premature convergence, as can occur in the worst-crowded approach, and 2) the presence of these extreme solutions will reduce the chance of having spurious non-Pareto-optimal solutions (like point $\mathrm{E}$ in Fig. 3) to remain in the nondominated front, thereby enabling a more accurate computation of the nadir point. Moreover, since the intermediate portion of the Pareto-optimal front is not targeted in this approach, finding the extreme solutions is expected to be quicker than the original NSGA-II, especially for problems having a large number of objectives and involving computationally expensive function evaluation schemes. 


\section{NADIR POINT ESTIMATION PROCEDURE}

It is clear that an accurate estimation of the nadir point depends on how accurately the critical points can be found. For solving multiobjective optimization problems, the NSGA-II approach (and for this matter any other EMO approach) is usually observed to find solutions near the Pareto-optimal front of a problem rather quickly and then reported to take many generations to reach arbitrarily close to the exact front [25]. Thus, to accurately find solutions on the Pareto-optimal front, NSGA-II solutions can be improved by using a local search approach [18], [26]. Likewise, for estimating the nadir point accurately, we propose to employ an EMO-cum-local-search approach, in which the solutions obtained by the modified NSGA-II approaches discussed above are improved by using a local-search procedure.

\section{A. Bilevel Local Search Approach}

Recall that due to the focus of the modified NSGA-II approaches toward individual objective-wise worst or extreme solutions, the algorithms are likely to find solutions close to the critical point for each objective. Therefore, the task of the proposed local search would be to then take each of these solutions to the corresponding critical point as accurately as possible. Particularly we would like to have the following three goals in our local search approach. First, the approach must be generic, so that it, for example, is applicable to convex and nonconvex problems alike. Second, the approach must guarantee convergence to the Pareto-optimal point, no matter which solutions are found by the modified NSGA-II approach. Third, the approach must find that particular Paretooptimal solution which corresponds to the worst value of the underlying objective. It is clear that the above task of the local search procedure involves two optimization tasks (to ensure the second task of finding a Pareto-optimal point and the third task of finding the worst objective-wise critical point, respectively). Unfortunately, both optimization tasks cannot be achieved through a single optimization procedure. In fact, both these problems form a bilevel optimization problem in which the upper level problem handles the second issue of finding the critical point, and a feasible solution of the upper level optimization problem must be an optimal solution to the lower-level problem (meaning a Pareto-optimal solution). In this sense, the proposed bilevel local search approach is different and more involved than the usual local search methods employed in EMO studies.

The first two goals mentioned above can be achieved by using a well-known MCDM approach, called the augmented achievement scalarizing function approach [1], [17]. In this approach, a reference point $\mathbf{z}$ is first chosen. By using a weight vector $\mathbf{w}$ (used for scaling), the following minimization problem is then solved:

$$
\begin{array}{ll}
\text { minimize } & \max _{j=1}^{M} w_{j}\left(f_{j}(\mathbf{x})-z_{j}\right)+\rho \sum_{j=1}^{M} w_{j}\left(f_{j}(\mathbf{x})-z_{j}\right) \\
\text { subject to } & \mathbf{x} \in \mathcal{S}
\end{array}
$$

where $\mathcal{S}$ is the original set of feasible solutions. The rightmost augmented term in the objective function is added so

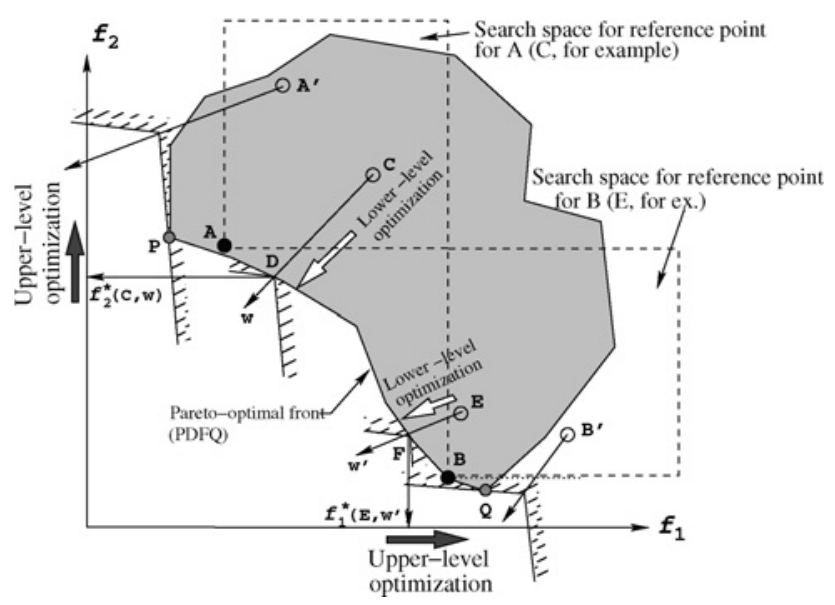

Fig. 5. Bilevel local search procedure is illustrated. A and B are worst objective-wise nondominated points obtained by EMO. The task of local search is to find critical point P from A and Q from B to make an accurate estimate of the nadir point.

that a weak Pareto-optimal solution (see, for example, [1] for a definition) is not found. For this purpose, a small value of $\rho$ (e.g., $10^{-4}$ or smaller) is used. The above optimization task involves a non-differentiable objective function (due to the max-term in the objective function), but if the original problem is differentiable, a suitable transformation of the problem can be made by introducing an additional slack variable $x_{n+1}$ to make an equivalent differentiable problem [1], as follows:

$$
\begin{aligned}
\text { minimize } & x_{n+1}+\rho \sum_{j=1}^{M} w_{j}\left(f_{j}(\mathbf{x})-z_{j}\right) \\
\text { subject to } & x_{n+1} \geq w_{j}\left(f_{j}(\mathbf{x})-z_{j}\right) \quad j=1,2, \ldots, M . \\
& \mathbf{x} \in \mathcal{S}
\end{aligned}
$$

If the single-objective optimization algorithm used to solve the above problem is able to find the true optimum, the optimal solution is guaranteed to be a Pareto-optimal solution [1]. In other words, achievement scalarizing functions project the reference point on the Pareto-optimal front. Moreover, the above approach is applicable for both convex and nonconvex problems. Fig. 5 illustrates the idea. For the reference point $\mathrm{C}$, the optimal solution of the above problem is $\mathrm{D}$, which is a Pareto-optimal point. The direction marked by the arrow depends on the chosen weight vector w. Irrespective of whether the reference point is feasible or not, the approach always finds a Pareto-optimal point dictated by the chosen weight vector and the reference point. The effect of the augmented term (with the term involving $\rho$ ) is shown by plotting a sketch of the iso-preference contour lines. More information about the role of weights is given, for example, in [27].

However, we also have a third goal of arriving at the objective-wise critical point. Thus, a task of finding any arbitrary Pareto-optimal solution is not adequate here, instead the aim of our local search procedure is to find the critical point corresponding to the underlying objective (like the point $\mathrm{P}$ for objective $f_{2}$ in Fig. 5). Unfortunately, it is not obvious which reference point and weight vector one must choose to arrive at a critical point. For this purpose, we construct another optimization problem to determine a combination of 
a reference point and a weight vector which will result in the critical point for an objective. This requires a nested bilevel approach in which the upper-level optimization considers a combination of a reference point and a weight vector $(\mathbf{z}, \mathbf{w})$ as decision variables. Each combination $(\mathbf{z}, \mathbf{w})$ is then evaluated by finding a Pareto-optimal solution corresponding to a lowerlevel optimization problem constructed using an augmented achievement scalarizing function given in (3) or (4) with $\mathbf{z}$ and $\mathbf{w}$ as the reference point and the weight vector, respectively. In the lower-level optimization, problem variables $(\mathbf{x})$ are the decision variables. As discussed above, the resulting optimal solution of the lower-level optimization is always a Paretooptimal solution (having an objective vector $\mathbf{f}^{*}$ ). Since our goal in the local search approach is to reach the critical point corresponding to a particular objective (say $j$ th objective), a solution $(\mathbf{z}, \mathbf{w})$ for the upper-level optimization task can be evaluated by checking the $j$ th objective value $\left(f_{j}^{*}\right)$ of the obtained Pareto-optimal solution.

Fig. 5 further explains this bilevel approach. Consider points $\mathrm{A}$ and $\mathrm{B}$ which are found by one of the modified NSGA-II procedures as worst objective-wise nondominated solutions for $f_{2}$ and $f_{1}$, respectively. The goal of using the local search approach is to reach the corresponding critical points ( $\mathrm{P}$ and $\mathrm{Q}$, respectively) from each of these points. Consider point $\mathrm{A}$, which is found to be the worst in objective $f_{2}$ among all modified NSGA-II solutions. The search region for the reference point $\mathbf{z}$ in the upper-level optimization is shown by the dashed box for which $\mathrm{A}$ is the lower-left corner point. Each component of the weight vector $(\mathbf{w})$ is restricted within a non-negative range of values $([0.001,1.000]$ is chosen for this paper). For the reference point $\mathbf{z}$, say $\mathrm{C}$, and weight vector $\mathbf{w}$ (directions indicating improvement of achievement scalarizing function), the solution to the lower-level optimization problem [problem (3) or (4)] is the decision variable vector $\mathbf{x}$ corresponding to solution D. Thus, for the reference point $\mathrm{C}$ and the chosen weight vector $(\mathbf{w})$, the corresponding function value of the upper-level optimization problem is the objective value $f_{2}$ of $\mathrm{D}$ (marked as $f_{2}^{*}(C, w)$ in the figure). Since this objective value is always computed for a Pareto-optimal solution (hence the * in its notation) and the upper-level optimization attempts to maximize this objective value iteratively, intuitively, the proposed bilevel local search approach is expected to find the critical point $\mathrm{P}$ (for $f_{2}$ ). It is interesting to note that there may exist many combinations of $(\mathbf{z}, \mathbf{w})$ (for example, with reference point $\mathrm{A}^{\prime}$ and weight vector shown by the arrow in the figure) which will also result in the same point $\mathrm{P}$ and for our purpose any one of such solutions would be adequate to accurately estimate the nadir point. Similarly, for the modified NSGA-II solution B (worst $f_{1}$ solution of NSGA-II), the critical point $\mathrm{Q}$ is expected to be the outcome of the above bilevel optimization approach. This critical point may result from many combinations of reference point and weight vectors (for example, from the reference point $\mathrm{B}^{\prime}$ and the weight vector shown by an arrow in the figure). In the bilevel approach, since we solve the single-objective lower-level problem [(3) or (4)] with an appropriate local optimization algorithm and the task of the upper-level search is also restricted in a local neighborhood by fixing variable bounds, we refer to this bilevel optimization approach as a local search algorithm here.

Now we are ready to outline the overall nadir point estimation procedure in a step-by-step format.

1) Step 1: Supply or compute ideal and worst objective vectors by minimizing and maximizing each objective function independently within the set of feasible solutions.

2) Step 2: Apply the worst-crowded or the extremizedcrowded NSGA-II approach to find a set of nondominated points. Iterations are continued until a termination criterion (described in the next subsection), which uses ideal and worst objective vectors computed in Step 1, is met. Say, $P$ nondominated extreme points (variable vector $\mathbf{x}_{\mathrm{EA}}^{(i)}$ with objective vector $\mathbf{f}_{\mathrm{EA}}^{(i)}$ for $i=1,2, \ldots, P$ ) are found in this step. Form the minimum and maximum objective vectors ( $\mathbf{f}^{\mathrm{min}}$ and $\mathbf{f}^{\max }$ ) from the $P$ obtained extreme solutions. For the $j$ th objective, they are computed as follows:

$$
\begin{aligned}
f_{j}^{\min } & =\min _{i=1}^{P} f_{j}^{(i)} \mathrm{EA} \\
f_{j}^{\max } & =\max _{i=1}^{P} f_{j}^{(i)} \mathrm{EA} .
\end{aligned}
$$

3) Step 3: Apply the bilevel local search approach for each objective $j(\in\{1, \ldots, M\})$, one at a time. First, identify the objective-wise worst solution (solution $\mathbf{x}_{\mathrm{EA}}^{(j)}$ for which the $j$ th objective has the worst value in $P$ ) and then find the corresponding optimal solution $\mathbf{y}^{*(j)}$ in the variable space by using the bilevel local search procedure, as follows. The upper-level optimization uses a combination of a reference point and a weight vector $(\mathbf{z}, \mathbf{w})$ as decision variables and maximizes the $j$ th objective value of the Pareto-optimal solution obtained by the lower-level optimization task (described a little later)

$$
\begin{aligned}
\operatorname{maximize}_{(\mathbf{z}, \mathbf{w})} & f_{j}^{*}(\mathbf{z}, \mathbf{w}) \\
\operatorname{subject~to~} & 0.001 \leq w_{j} \leq 1, \quad j=1,2, \ldots, M \\
& z_{i} \geq f_{i}^{(j)} \mathrm{EA} \quad i=1,2, \ldots, M \\
& z_{i} \leq f_{i}^{(j)} \mathrm{EA}+\left(f_{i}^{\max }-f_{i}^{\mathrm{min}}\right) \\
& i=1,2, \ldots, M .
\end{aligned}
$$

The term $f_{j}^{*}(\mathbf{z}, \mathbf{w})$ is the value of the $j$ th objective function at the optimal solution to the following lower-level optimization problem:

$$
\begin{array}{lc}
\operatorname{minimize}_{(\mathbf{y})} & \max _{i=1}^{M} w_{i}\left(\frac{f_{i}(\mathbf{y})-z_{i}}{f_{i}^{\max }-f_{i}^{\text {min }}}\right) \\
& +\rho \sum_{k=1}^{M} w_{k}\left(\frac{f_{k}(\mathbf{y})-z_{k}}{f_{k}^{\max }-f_{k}^{\min }}\right) \\
\text { subject to } & \mathbf{y} \in \mathcal{S} .
\end{array}
$$

This problem is identical to that in (3), except that individual objective terms are normalized for a better property of the augmented term. In this lower-level optimization problem, the search is performed on the original decision variable space. The solution $\mathbf{y}^{*(j)}$ to this lower-level optimization problem determines the optimal objective vector $\mathbf{f}\left(\mathbf{y}^{*(j)}\right)$ from which we extract the $j$ th component and use it as the objective value 
for the upper-level solution $(\mathbf{z}, \mathbf{w})$. Thus, for every reference point $\mathbf{z}$ and weight vector $\mathbf{w}$, considered in the upper-level optimization task, the corresponding optimal augmented achievement scalarizing function is found by solving the lower-level optimization problem. The upper-level optimization is initialized with the NSGA-II solution $\mathbf{z}^{(0)}=\mathbf{f}\left(\mathbf{x}_{\mathrm{EA}}^{(j)}\right)$ and $w_{i}^{(0)}=1 / M$. The lower-level optimization is initialized with the NSGA-II solution $\mathbf{y}^{(0)}=\mathbf{x}_{\text {EA }}^{(j)}$. The local search can be terminated based on standard single-objective convergence measures, such as Karush-Kuhn-Tucker (KKT) condition satisfaction through a prescribed limit or a small difference in variable vectors between successive iterations.

4) Step 4: Finally, construct the nadir point from the worst objective values of the all Pareto-optimal solutions obtained after the local search procedure.

The use of a bilevel local search approach can be computationally expensive, if the starting solution to the local search is far away from the critical point. For this reason, the proposed local search procedure may not be computationally viable if started from a random initial point. However, the use of a modified NSGA-II approach to first find a near critical point and then to employ the proposed local search to accurately locate the critical point seems like a viable approach. To demonstrate the computational viability of using the proposed local search approach within our nadir point estimation procedure, we shall present a break-up of function evaluations needed by both NSGA-II and local search procedures later.

Before we leave this subsection, we discuss one further issue. It is mentioned above that the use of the augmentation term in the achievement scalarizing problem formulation allows us not to converge to a weakly Pareto-optimal solution by the local search approach. But, in certain problems, the approach may only find a critical proper Pareto-optimal solution [1] depending on the value of the parameter $\rho$. For this reason, we actually get an estimate of the ranges of objective function values in a properly Pareto-optimal set and not in a Pareto-optimal set. We can control the trade-offs in the properly Pareto-optimal set by choosing an appropriately small $\rho$ value. For further details, see, for example, [1]. In certain problems having a small trade-off near the critical points, a proper Pareto-optimal point can be somewhat away from the true critical point. If this is not desired, it is possible to solve a lexicographic achievement scalarizing function [1], [2] instead of the augmented one suggested in Step 3.

\section{B. Termination Criterion for Modified NSGA-II}

Typically, a NSGA-II run is terminated when a pre-specified number of generations is elapsed. Here, we suggest a performance based termination criterion which causes a NSGA-II run to stop when the performance reaches a desirable level. The performance metric depends on a measure stating how close the estimated nadir point is to the true nadir point. However, for applying the proposed NSGA-II approaches to an arbitrary problem (for which the true Pareto-optimal front, hence the true nadir point, is not known a priori), we need a different concept. Using the ideal point $\left(\mathbf{z}^{*}\right)$, the worst objective vector $\left(\mathbf{z}^{w}\right)$, and the estimated nadir point (to be denoted as $\mathbf{z}^{\text {est }}$ ) at any generation of NSGA-II, we can define a normalized distance (ND) metric as follows and track the convergence property of this metric to determine the termination of our NSGA-II approach:

$$
N D=\sqrt{\frac{1}{M} \sum_{i=1}^{M}\left(\frac{z_{i}^{\mathrm{est}}-z_{i}^{*}}{z_{i}^{w}-z_{i}^{*}}\right)^{2} .}
$$

If in a problem, the worst objective vector $\mathbf{z}^{w}$ (refer to Fig. 1) is the same as the nadir point, the $N D$ metric value must converge to one. Since the exact final value of this metric for finding the true nadir point is not known a priori on an arbitrary problem, we record the change in $N D$ for the past $\tau$ generations. Let us now denote $N D_{\max }, N D_{\min }$, and $N D_{\text {avg }}$, as the maximum, minimum, and average $N D$ values for the past consecutive $\tau$ generations. If the normalized change $\left(N D_{\max }-\right.$ $\left.N D_{\text {min }}\right) / N D_{\text {avg }}$ is smaller than a threshold $\Delta$, the proposed NSGA-II approach is terminated and the current nondominated extreme solutions are sent to the next step for performing the local search.

However, in the case of solving test problems, the location of the nadir objective vector is expected to be known and a simple error metric $(E)$ between the estimated and the known nadir objective vectors can be used for stopping a NSGA-II run to investigate the working of our proposed procedure

$$
E=\sqrt{\sum_{i=1}^{M}\left(\frac{z_{i}^{\mathrm{nad}}-z_{i}^{\mathrm{est}}}{z_{i}^{\mathrm{nad}}-z_{i}^{*}}\right)^{2} .}
$$

To make the approach pragmatic, in this paper, we terminate a NSGA-II run when the error metric $E$ becomes smaller than a predefined threshold $(\eta)$.

\section{RESUltS ON BENCHMARK PROBLEMS}

We are now ready to describe the results of numerical tests obtained using the proposed hybrid nadir point estimation procedure. We have chosen problems having three to 20 objectives in this paper. In this section, we use benchmark problems where the entire description of the objective space and the Pareto-optimal front is known. We have chosen these problems to test the working of our procedure. Thus, in these problems, we do not perform Step 1 explicitly. Moreover, if Step 2 of the nadir point estimation procedure successfully finds the nadir point (using the error metric $(E \leq \eta)$ for determining termination of a run), we do not employ Step 3 (local search). The complete hybrid procedure will be tested in its totality in the next section.

In all runs here, we compare three different approaches:

1) naive NSGA-II approach in which first we find a set of nondominated solutions using the original NSGA-II and then estimate the nadir point from the obtained solutions;

2) NSGA-II with the worst-crowded approach;

3) NSGA-II with the extremized-crowded approach.

To make a fair comparison, parameters in all three cases are kept fixed for all problems. We use the simulated binary crossover (SBX) recombination operator [28] with a probability of 0.9 and a distribution index of $\eta_{c}=10$. The polynomial 
mutation operator [18] is used with a probability of $1 / n$ ( $n$ is the number of variables) and a distribution index of $\eta_{m}=20$. The population size is set according to the problem and is mentioned in respective subsections. Each algorithm is run 11 times (odd number of runs is used to facilitate the recording of the median performance of an algorithm), each time starting from a different random initial population. However all proposed procedures are started with an identical set of initial populations to be fair. The number of generations required to satisfy the termination criterion $(E \leq \eta)$ is noted for each run and the corresponding best, median, and worst number of generations are presented for a comparison. For all test problems, $\eta=0.01$ is used.

\section{A. Three and More Objectives}

To test Step 2 of the nadir point estimation procedure on three and more objectives, we choose three Deb, Thiele, Laumanns and Zitzler (DTLZ) test problems [29] which have different characteristics. These problems are designed in a manner so that they can be extended to any number of objectives. The first problem, DTLZ1, is constructed to have a linear Pareto-optimal front. The true nadir objective vector is $\mathbf{z}^{\mathrm{nad}}=(0.5, \ldots, 0.5)^{T}$ and the ideal objective vector is $\mathbf{z}^{*}=(0, \ldots, 0)^{T}$. The Pareto-optimal front of the second test problem, DTLZ2, is a quadrant of a unit sphere centered at the origin of the objective space. The nadir objective vector is $\mathbf{z}^{\text {nad }}=(1, \ldots, 1)^{T}$ and the ideal objective vector is $\mathbf{z}^{*}=$ $(0, \ldots, 0)^{T}$. The third test problem, DTLZ5, is somewhat modified from the original DTLZ5 and has a 1-D Pareto-optimal curve in the $M$-dimensional space [21]. The ideal objective vector is $\mathbf{z}^{*}=(0, \ldots, 0)^{T}$ and the nadir objective vector is $\mathbf{z}^{\mathrm{nad}}=\left(\left(\frac{1}{\sqrt{2}}\right)^{M-2},\left(\frac{1}{\sqrt{2}}\right)^{M-2},\left(\frac{1}{\sqrt{2}}\right)^{M-3}, \quad\left(\frac{1}{\sqrt{2}}\right)^{M-4}, \ldots,\left(\frac{1}{\sqrt{2}}\right)^{0}\right)^{T}$.

1) Three-Objective DTLZ Problems: All three approaches are run with 100 population members for problems DTLZ1, DTLZ2, and DTLZ5 involving three objectives. Table I shows the numbers of generations needed to find a solution close (within an error metric value of $\eta=0.01$ or smaller) to the true nadir point. It can be observed that the worst-crowded NSGA-II and the extremized-crowded NSGA-II perform in a more or less similar way when compared to each other and are somewhat better than the naive NSGA-II approach. In the DTLZ5 problem, despite having three objectives, the Paretooptimal front is 1-D [29]. Thus, the naive NSGA-II approach performs almost as well as the proposed modified NSGA-II approaches.

To compare the working principles of the two modified NSGA-II approaches and the naive NSGA-II approach, we show the final populations for the extremized-crowded NSGA-II and the naive NSGA-II for DTLZ1 and DTLZ2 in Figs. 6 and 7, respectively. Similar results are also found for the worst-crowded NSGA-II approach, but are not shown here for brevity. It is clear that the extremized-crowded NSGA-II concentrates its population members near the extreme regions of the Pareto-optimal front, so that a quicker estimation of the nadir point is possible to achieve. However, in the case of the naive NSGA-II approach, a distributed set of Paretooptimal solutions is first found using the original NSGA-II

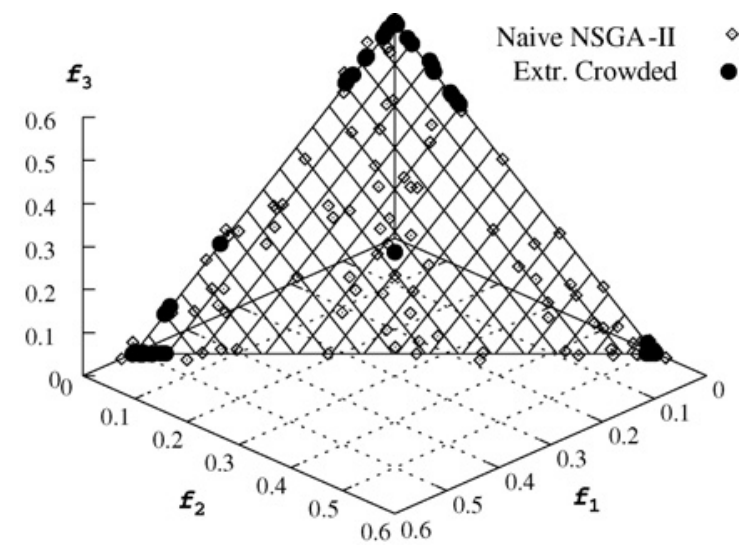

Fig. 6. Populations obtained using extremized-crowded and naive NSGA-II for DTLZ1. Extremized-crowded NSGA-II finds the objective-wise extreme points, whereas the naive NSGA-II approach finds a distributed set of points.

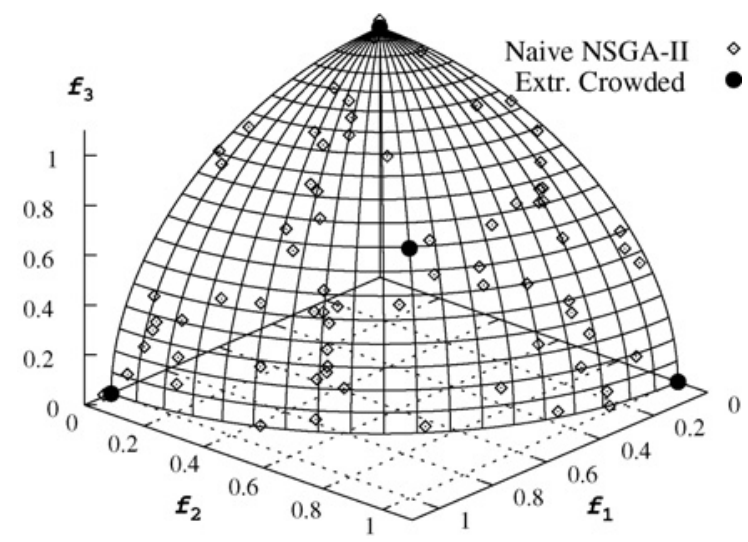

Fig. 7. Populations obtained using extremized-crowded and naive NSGA-II for DTLZ2. Extremized-crowded NSGA-II finds objective-wise extreme points.

(as shown in the figure) and the nadir point is constructed from these points. Since the intermediate points do not help in constructing the nadir objective vector, the naive NSGA-II approach is expected to be computationally inefficient and also comparatively inaccurate, particularly for problems having a large number of objectives. There is not much of a difference in the performance of the original NSGA-II and modified NSGA-IIs for DTLZ5 problem due to the 1-D nature of the Pareto-optimal front. Hence, we do not show the corresponding figure here.

To investigate if the error metric $(E)$ reduces with generations, we continue to run the two modified NSGA-II procedures till 1000 generations. For the DTLZ1 problem, the worst-crowded approach settles on an $E$ value in the range $[0.000200,0.000283]$ for 11 independent runs and the extremized-crowded approach in the range [0.000199, 0.000283]. For DTLZ2, both approaches settle to $E=$ 0.000173 and for DTLZ5, worst-crowded and extremizedcrowded NSGA-IIs settle in the range [0.000211, 0.000768] and [0.000211, 0.000592], respectively. Since a threshold of $E \leq 0.01$ was used for termination in obtaining results in Table I, respective NSGA-IIs terminated at a generation smaller than 1000 . However, these results show that there is no 
TABLE I

COMPARATIVE RESUlts FOR DTLZ PRoBlems With THREE OBJECTIVES

\begin{tabular}{|c|c|c|c|c|c|c|c|c|c|c|}
\hline Test & Pop. & \multicolumn{9}{|c|}{ Number of Generations } \\
\cline { 3 - 11 } Problem & \multirow{2}{*}{ Size } & \multicolumn{3}{|c|}{ NSGA-II } & \multicolumn{2}{|c|}{ Worst-Crowded NSGA-II } & \multicolumn{2}{c|}{ Extremized-Crowded NSGA-II } \\
\cline { 3 - 11 } & & Best & Median & Worst & Best & Median & Worst & Best & Median & Worst \\
\hline DTLZ1 & 100 & 223 & 366 & 610 & 171 & 282 & 345 & 188 & 265 & 457 \\
\hline DTLZ2 & 100 & 75 & 111 & 151 & 38 & 47 & 54 & 41 & 49 & 55 \\
\hline DTLZ5 & 100 & 63 & 80 & 104 & 59 & 74 & 86 & 62 & 73 & 88 \\
\hline
\end{tabular}

significant change in the nadir point estimation with the extra computations and the proposed procedure has a convergent property (which will also be demonstrated on higher objective problems through convergence metrics of this paper in Figs. 8$10,13,15$, and 17).

2) Five-Objective DTLZ Problems: Next, we study the performance of all three NSGA-II approaches on DTLZ problems involving five objectives. In Table II, we collect information about the results as in the previous subsection. It is now quite evident from Table II that the modifications proposed to the NSGA-II approach perform much better than the naive NSGA-II approach. For example, for the DTLZ1 problem, the best NSGA-II run takes 2342 generations to estimate the nadir point, whereas the extremized-crowded NSGA-II requires only 353 generations and the worst-crowded NSGAII 611 generations. In the case of the DTLZ2 problem, the trend is similar. The median generation counts of the modified NSGA-II approaches for 11 independent runs are also much better than those of the naive NSGA-II approach.

The difference between the worst-crowded and extremizedcrowded NSGA-II approaches is also clear from the table. For a problem having a large number of objectives, the extremizedcrowded NSGA-II emphasizes both best and worst extreme solutions for each objective maintaining an adequate diversity among the population members. The genetic operators are able to exploit a relatively diversified population and make a faster progress toward the extreme Pareto-optimal solutions needed to estimate the nadir point correctly. However, on the DTLZ5 problem, the performance of all three approaches is similar due to the 1-D nature of the Pareto-optimal front. Fig. 8 shows the convergence of the error metric value for the best runs of the three algorithms on DTLZ2. The figure demonstrates the convergent property of the proposed algorithm. The superiority of the extremized-crowded NSGA-II approach is clear from the figure. Similar results are also observed for DTLZ1. These results imply that for a problem having more than three objectives, an emphasis on the extreme Pareto-optimal solutions (instead of all Pareto-optimal solutions) is a faster approach for locating the nadir point.

So far, we have demonstrated the ability of the nadir point estimation procedure in converging close to the nadir point by tracking the error metric value which requires the knowledge of the true nadir point. It is clear that this metric cannot be used in an arbitrary problem. We have suggested a $N D$ metric (9) for this purpose. To demonstrate how the $N D$ metric can be used as a termination criterion, we record this metric value at every generation for both extremized-crowded NSGA-II and the naive NSGA-II runs and plot them in Fig. 9 for

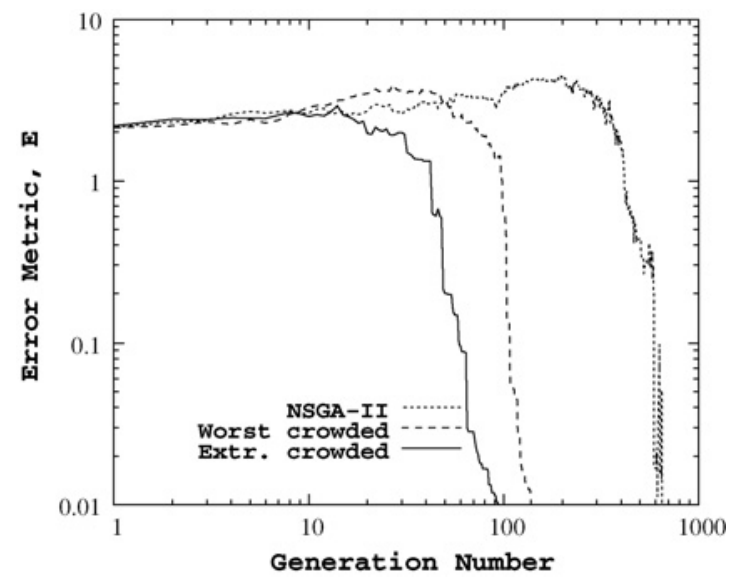

Fig. 8. Error metric for best of 11 runs on five-objective DTLZ2. Extremized-crowded NSGA-II is about an order of magnitude better than the naive NSGA-II approach.

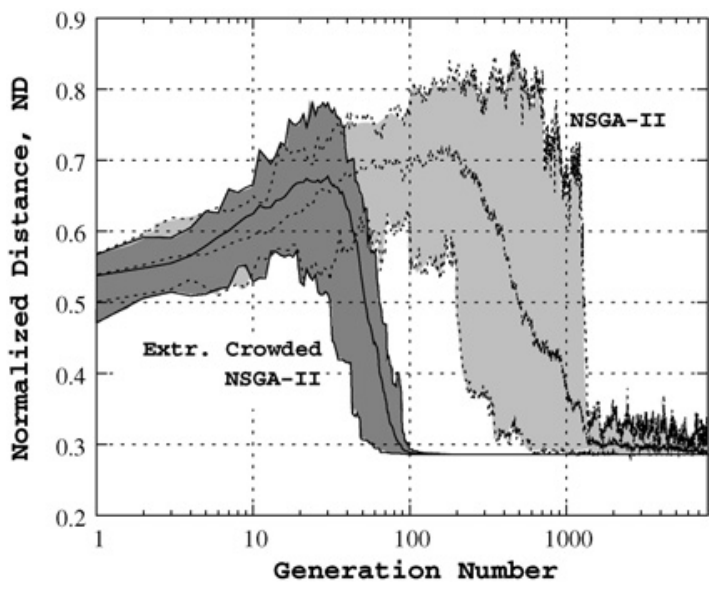

Fig. 9. Variation of $N D$ metric in 11 runs for two methods on five-objective DTLZ2. Extremized-crowded NSGA-II is about an order of magnitude better than the naive NSGA-II approach.

DTLZ2. Similar trends were observed for the worst-crowded NSGA-II and also for test problem DTLZ1, but for brevity these results are not shown here. To show the variation of the metric value over different initial populations, the region between the best and the worst $N D$ metric values is shaded and the median value is shown with a line. Recall that the $N D$ metric requires the information of the worst objective vector $\left(\mathbf{z}^{w}\right)$. For the DTLZ2 problem, the worst objective vector is found to be $z_{i}^{w}=3.25$ for $i=1, \ldots, 5$. Fig. 9 shows that the $N D$ metric $(N D)$ value converges to around 0.286 , which is identical to that computed by substituting the estimated nadir 
TABLE II

Comparative Results For Five AND Ten-OBJective DTLZ Problems

\begin{tabular}{|c|c|c|c|c|c|c|c|c|c|c|}
\hline \multirow{3}{*}{$\begin{array}{c}\text { Test } \\
\text { Problem }\end{array}$} & \multirow{3}{*}{$\begin{array}{l}\text { Pop. } \\
\text { Size }\end{array}$} & \multicolumn{9}{|c|}{ Number of Generations } \\
\hline & & \multicolumn{3}{|c|}{ NSGA-II } & \multicolumn{3}{|c|}{ Worst-Crowded NSGA-II } & \multicolumn{3}{|c|}{ Extremized-Crowded NSGA-II } \\
\hline & & Best & Median & Worst & Best & Median & Worst & Best & Median & Worst \\
\hline & & \multicolumn{9}{|c|}{ Five-Objective DTLZ Problems } \\
\hline DTLZ1 & 100 & 2342 & 3136 & 3714 & 611 & 790 & 1027 & 353 & 584 & 1071 \\
\hline DTLZ2 & 100 & 650 & 2142 & 5937 & 139 & 166 & 185 & 94 & 114 & 142 \\
\hline \multirow[t]{2}{*}{ DTLZ5 } & 100 & 52 & 66 & 77 & 51 & 66 & 76 & 49 & 61 & 73 \\
\hline & & \multicolumn{9}{|c|}{ Ten-Objective DTLZ Problems } \\
\hline DTLZ1 & 200 & 17581 & 21484 & 33977 & 1403 & 1760 & 2540 & 1199 & 1371 & 1790 \\
\hline DTLZ2 & 200 & - & - & - & 520 & 823 & 1456 & 388 & 464 & 640 \\
\hline DTLZ5 & 200 & 45 & 53 & 60 & 43 & 53 & 57 & 45 & 51 & 64 \\
\hline
\end{tabular}

objective vector with the true nadir objective vector in (9). Thus, we can conclude that the convergence of the extremizedcrowded NSGA-II is on the true nadir point. Despite the large variability in $N D$ value in different runs early on, all 11 runs of the extremized-crowded NSGA-II finally converge to the critical points at around 100 generations without much variance, indicating the robustness of the procedure. Similarity of this convergence pattern (at generation 100) with the fast convergence demonstrated in Fig. 8 at around 100 generation indicates that the $N D$ metric (using ideal and worst objective vectors) signifies a similar convergence to the nadir point as that obtained with the exact nadir and ideal objective vectors used in the error metric. Hence, the $N D$ metric can be used in arbitrary problems. A fast rate of convergence is also interesting to note from Fig. 9. The extremized-crowded NSGA-II is able to find the nadir point much quicker (almost an order of magnitude faster) than the naive NSGA-II approach. Due to clear and visible demonstration of superiority of the extremized-crowded NSGA-II through these figures, we do not perform any further statistical tests.

3) Ten-Objective DTLZ Problems: Next, we consider the three DTLZ problems for ten objectives. Due to the increase in the dimensionality of the objective space, we double the population size for these problems. Table II presents the numbers of generations required to find a point close (within $\eta=0.01)$ to the nadir point by the three approaches for the DTLZ problems with ten objectives. It is clear that the extremized-crowded NSGA-II approach performs an order of magnitude better than the naive NSGA-II approach and is also better than the worst crowded NSGA-II approach. Both the DTLZ1 and DTLZ2 problems have 10-D Pareto-optimal fronts and the extremized-crowded NSGA-II makes a good balance of maintaining diversity and emphasizing extreme Pareto-optimal solutions so that the nadir point estimation is quick. In the case of the DTLZ2 problem with ten objectives, the naive NSGA-II could not find the nadir objective vector even after 50000 generations (and achieved an error metric value of 5.936). Fig. 10 shows a typical convergence pattern of the extremized-crowded NSGA-II and the naive NSGA-II approaches on the ten-objective DTLZ1 problem.

The figure demonstrates that for a large number of generations the estimated nadir point is far away from the true

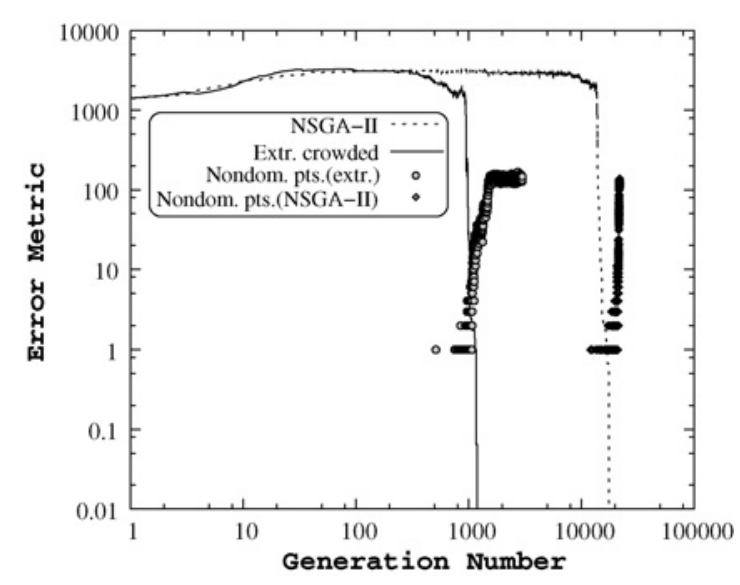

Fig. 10. Performance of two methods on ten-objective DTLZ1. Extremizedcrowded NSGA-II is about an order of magnitude better than the naive NSGAII approach. Convergence becomes faster after a solution dominating the nadir point is discovered.

nadir point, but after some generations (around 1000 in this problem) the estimated nadir point comes quickly near the true nadir point. To understand the dynamics of the movement of the population in an extremized-crowded NSGA-II simulation with the generation counter, we count the number of population members which dominate the true nadir point and plot this quantity in Fig. 10. Points which dominate the nadir point lie in the region between the Pareto-optimal front and the nadir point. Thus, a task of finding these points is important toward reaching the critical points and therefore in estimating the nadir point. It is extremely unlikely to create such important points at random, particularly when dealing with a large number of objectives. Thus, an optimization algorithm, starting with random solutions, must work toward finding such important points first before converging to the Pareto-optimal front. In DTLZ1, it is seen that the first point dominating the true nadir point appears in the population at around 750 generations with the extremized-crowded approach, whereas the naive NSGA-II needed about 10000 generations. Thereafter, when an adequate number of such solutions start appearing in the population, the population very quickly converges near the critical points for correctly estimating the nadir point. 


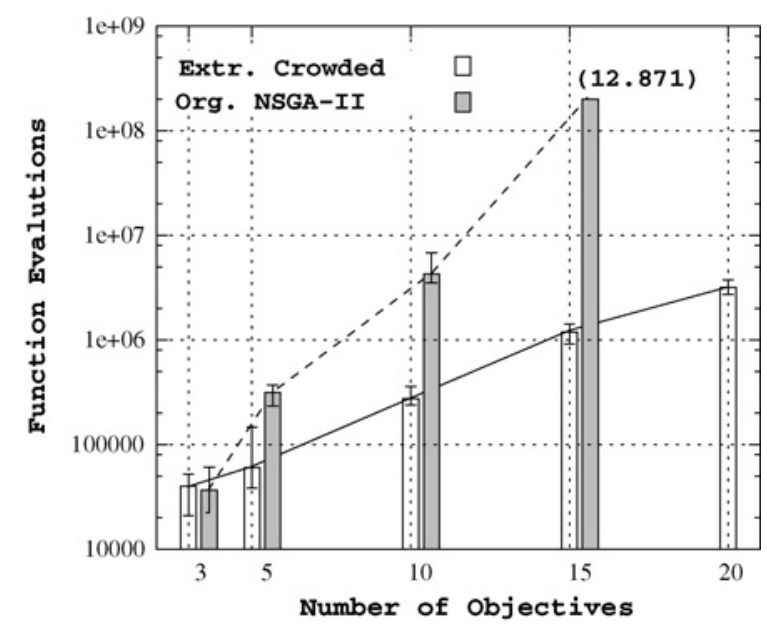

Fig. 11. Function evaluations versus number of objectives for DTLZ1.

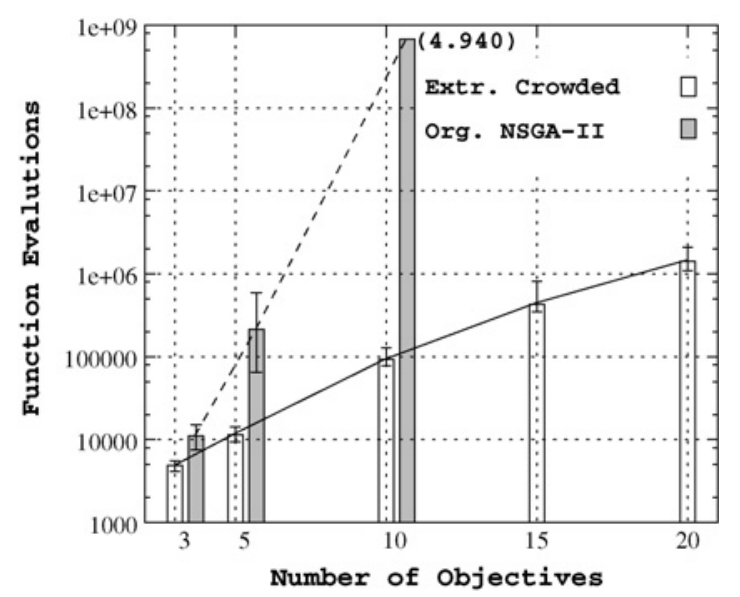

Fig. 12. Function evaluations versus number of objectives for DTLZ2.

\section{B. Scale-Up Performance}

Let us next investigate the overall function evaluations required to get near the true nadir point on DTLZ1 and DTLZ2 test problems having three to 20 objectives. As before, we use the stopping criterion $E \leq 0.01$. Here, we investigate the scale-up performance of the extremized-crowded NSGA-II alone and compare it against that of the naive NSGA-II approach. Since the worst-crowded NSGA-II did not perform well on ten-objective DTLZ problems compared to the extremized-crowded NSGA-II approach, we do not consider it here.

Fig. 11 plots the best, median, and worst of 11 runs of the extremized-crowded NSGA-II and the naive NSGA-II on DTLZ1. First of all, the figure clearly shows that the naive NSGA-II is unable to scale up to 15 or 20 objectives. In the case of 15-objective DTLZ1, the naive NSGA-II's performance is more than two orders of magnitude worse than that of the extremized-crowded NSGA-II. For this problem, the naive NSGA-II with more than 200 million function evaluations obtained a front having a poor error metric value of 12.871 . Due to the poor performance of the naive NSGA-II approach on the 15-objective problem, we did not apply it to the 20objective DTLZ1 problem.
Fig. 12 shows the performances on DTLZ2. After 670 million function evaluations, the naive NSGA-II was still not able to come close (with an error metric value of 0.01) to the true nadir point on the ten-objective DTLZ2 problem. However, the extremized-crowded NSGA-II took an average of 99000 evaluations to achieve the task. Because of the computational inefficiencies associated with the naive NSGA-II approach, we did not perform any runs for 15 or more objectives, whereas the extremized-crowded NSGA-II could find the nadir point up to the 20-objective DTLZ2 problem.

The nature of the plots for the extremized-crowded NSGA-II in both problems is found to be sub-linear on a semilogarithmic plot. This indicates a lower than exponential scaling property of the proposed extremized-crowded NSGA-II. It is important to emphasize here that estimating the nadir point requires identification of the critical points. Since this requires that an evolutionary approach essentially puts its population members on the Pareto-optimal front, an adequate computational effort must be spent to achieve this task. However, results shown earlier for three to ten-objective problems have indicated that the computational effort needed by the extremized-crowded NSGA-II approach is smaller when compared to the naive NSGA-II. It is worth pointing out here that decision makers do not necessarily want to or are not necessarily able to consider problems with very many objectives. However, the results of this paper show a clear difference even with smaller problems involving, for example, five objectives.

\section{Results of Tests With the FUll Hybrid NADIR POINT ESTIMATION PROCEDURE}

Now, we apply the complete hybrid nadir point estimation procedure which makes a serial application of the extremizedcrowded NSGA-II approach followed by the bilevel local search approach on three optimization problems. Since in the previous problems we identified difficulties with the worstcrowded NSGA-II, we do not continue with the worst-crowded NSGA-II procedure any more. The first two problems are numerical test problems taken from the MCDM literature on which the payoff table method is reported to have failed to estimate the nadir point accurately, and the third problem is a nonlinear engineering design problem. All these problems adequately demonstrate the usefulness of the proposed hybrid procedure with the extremized-crowded NSGA-II approach. For all problems of this section, we use a population size of $20 n$, where $n$ is the number of variables and keep other NSGA-II parameters as they were used in the previous section. For both upper and lower-level optimizations in the local search, we have used the fmincon routine (implementing the sequential quadratic programming (SQP) method in which every approximated quadratic programming problem is solved using the Broyden-Fletcher-Goldfarb-Shanno quasi-Newton procedure) of MATLAB with default parameter values.

\section{A. Problem Klamroth-Miettinen (KM)}

We consider a three-objective optimization problem, which provides difficulty for the payoff table method to estimate the 
nadir point. This problem was used in [30]

$$
\begin{array}{cl}
\operatorname{minimize} & \left\{\begin{array}{l}
-x_{1}-x_{2}+5 \\
\frac{1}{5}\left(x_{1}^{2}-10 x_{1}+x_{2}^{2}-4 x_{2}+11\right) \\
\left(5-x_{1}\right)\left(x_{2}-11\right)
\end{array}\right\} \\
\text { subject to } & 3 x_{1}+x_{2}-12 \leq 0 \\
& 2 x_{1}+x_{2}-9 \leq 0 \\
& x_{1}+2 x_{2}-12 \leq 0 \\
& 0 \leq x_{1} \leq 4 \quad 0 \leq x_{2} \leq 6
\end{array}
$$

Individual minimizations of objectives reveal the following three objective vectors: $(-2,0,-18)^{T},(0,-3.1,-14.25)^{T}$, and $(5,2.2,-55)^{T}$, thereby identifying the ideal vector $\mathbf{z}^{*}=(-2,-3.1,-55)^{T}$. The payoff table method finds $(5,2.2,-14.25)^{T}$ as the estimated nadir point from these minimization results, which is a wrong estimate as discussed below. Another paper [31] used an exhaustive grid-search strategy (computationally possible due to having only two variables and three objectives in this problem) of creating a number of feasible solutions systematically and constructing the nadir point from the solutions obtained. Since an exhaustive search was used, we can say that the true nadir point of the problem is $(5,4.6,-14.25)^{T}$. We now employ our nadir point estimation procedure to investigate if it is able to find this true nadir point.

Step 1 of the procedure, described in Section IV-A, finds $\mathbf{z}^{*}=(-2,-3.1,-55)^{T}$ and $\mathbf{z}^{w}=(5,4.6,-14.25)^{T}$ by minimizing and maximizing each objective function individually.

In Step 2 of the procedure, we employ the extremizedcrowded NSGA-II. As a result, we obtain four different nondominated extreme solutions, as shown in the first column of Table III. The extremized-crowded NSGA-II approach is terminated when the $N D$ metric does not change by an amount $\Delta=0.0001$ in a consecutive $\tau=50$ generations. It is interesting to note that the fourth solution is not needed to estimate the nadir point, but the extremized principle keeps this extreme solution corresponding to $f_{1}$ to possibly eliminate spurious solutions which may otherwise stay in the population and provide a wrong estimate of the nadir point (see Fig. 3 for a discussion). Fig. 13 shows the variation of the $N D$ metric value with generation, computed using the above-mentioned ideal and worst objective vectors. The NSGA-II procedure was terminated at generation 135 , due to the fall of the $N D$ value below the chosen threshold of 0.0001 . At the end of Step 2, the estimated nadir point is $\mathbf{z}^{\text {nad }}=(5,4.6,-14.194)^{T}$, which seems to disagree on the third objective value with that found by the exhaustive grid-search strategy.

In Step 3, we now apply the bilevel local search approach from each of the four solutions presented in Table III, as they are found to be the extreme nondominated solutions using NSGA-II. The minimum and maximum objective vectors from these solutions are: $(-1,-3.1,-55)^{T}$ and $(5,4.6,-14.194)^{T}$, respectively. Recall that the local search method suggested here is a bilevel optimization procedure in which the upperlevel optimization uses a combination of a weight vector and a reference point as a decision variable vector $(\mathbf{z}, \mathbf{w})$ with an objective of maximizing the objective value for which the corresponding NSGA-II solution is the worst. The lower-

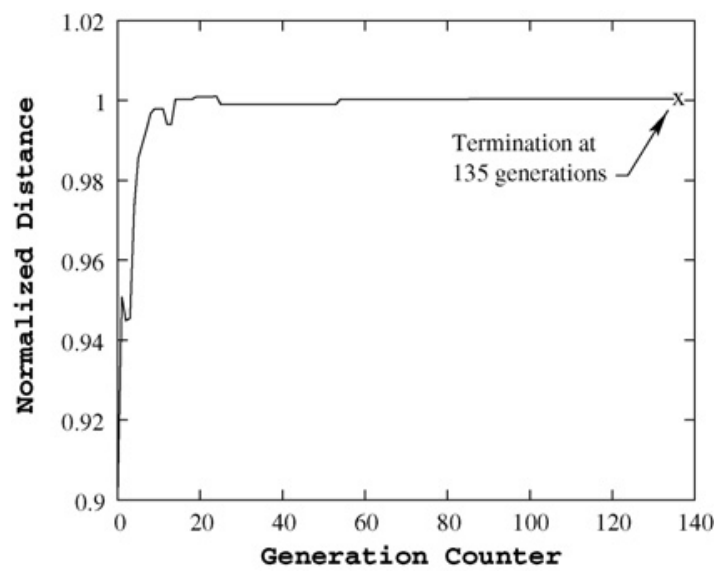

Fig. 13. Variation of $N D$ metric with generation for problem KM.

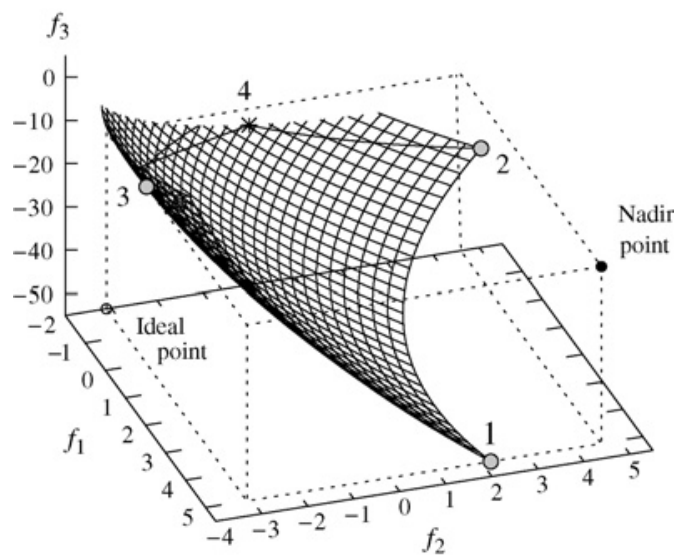

Fig. 14. Pareto-optimal front with extreme points for problem KM. Point 4 is best for $f_{1}$, but not worst for any objective. Thus, it is redundant for estimating the nadir point.

level optimization loop uses variable vector $\mathbf{x}$ and minimizes the corresponding achievement scalarizing function with $\rho=$ $10^{-5}$.

Solution 1 from Table III corresponds to the worst value of the first objective $\left(f_{1}\right)$. Thus, the upper-level optimization task maximizes objective $f_{1}$. Starting with the NSGA-II solution (column 2 in the table), the local search approach finds a solution shown in the sixth column. Since this particular NSGA-II solution happens to be truly the critical point for $f_{1}$, the local search terminates after two iterations and declares the same solution as the outcome of the local search.

Solution 2 has the worst value for objective $f_{3}$ among the four obtained NSGA-II solutions. Table III clearly shows that solution 2 (the objective vector $(0.023,-3.100,-14.194)^{T}$, obtained by the extremized-crowded NSGA-II), was close to the Pareto-optimal front, but was not a Pareto-optimal solution. However, the proposed local search approach starting from this solution is able to find a better solution $(0,-3.1,-14.25)^{T}$. This shows the importance of employing the local search in our hybrid approach.

Solution 3 has the worst value for objective $f_{2}$. The proposed local search approach does not improve this solution, as this is truly the critical point for $f_{3}$. 
TABLE III

EXTREMIZED-CRowded NSGA-II AND LOCAL SEARCH METHOd ON PROBLEM KM

\begin{tabular}{|c|c|c|c|c|c|}
\hline & $\mathbf{x}_{\text {NSGA-II }}$ & Objective Vector, $\mathbf{f}_{\text {NSGA-II }}$ & $\mathbf{w}$ & $\mathbf{z}$ & Extreme Point \\
\hline 1 & $(0,0)^{T}$ & $(5,2.2,-55)^{T}$ & $(0.333,0.333,0.333)^{T}$ & $(5,2.2,-55)^{T}$ & $(5,2.2,-55)^{T}$ \\
2 & $(3.511,1.466)^{T}$ & $(0.023,-3.100,-14.194)^{T}$ & $(0.335,0.335,0.334)^{T}$ & $(0.023,-3.085,-14.114)^{T}$ & $(0,-3.1,-14.25)^{T}$ \\
3 & $(0,6)^{T}$ & $(-1,4.6,-25)^{T}$ & $(0.333,0.333,0.333)^{T}$ & $(-1,4.6,-25)^{T}$ & $(-1,4.6,-25)^{T}$ \\
\cline { 4 - 6 } 4 & $(2.007,4.965)^{T}$ & $(-1.973,-0.050,-18.060)^{T}$ & \multicolumn{2}{|c|}{ Not worse in any objective, so not considered } \\
\hline
\end{tabular}

Solution 4 does not have the worst value for any of the objectives, so we do not perform a local search from this solution. Fig. 14 shows the Pareto-optimal front for this problem. These three extreme Pareto-optimal points are marked on the front with a shaded circle. The fourth point is also shown with a star.

Finally, in Step 4 of the proposed hybrid approach, the nadir point estimated by the combination of the extremizedcrowded NSGA-II and the bilevel local search approach is $(5,4.6,-14.25)^{T}$, which is identical to that obtained by the exhaustive grid search strategy [31]. As discussed earlier, the exhaustive grid search strategy is not scalable to largedimensional problems due to an exponential increase in computations.

The extremized-crowded NSGA-II approach took 5440 solution evaluations and the three local search runs took a total of 1583 solution evaluations, thereby requiring a total of 7023 solution evaluations. Thus, the NSGA-II approach needed a major share of the overall computational effort of about $77 \%$ and the bilevel local search approach took only about $23 \%$ of the total effort.

\section{B. Problem Szczepanski-Wierzbicki (SW)}

Next, we consider a linear problem presented in [13]

$$
\begin{array}{cl}
\text { minimize } & \left\{\begin{array}{l}
9 x_{1}+19.5 x_{2}+7.5 x_{3} \\
7 x_{1}+20 x_{2}+9 x_{3} \\
-\left(4 x_{1}+5 x_{2}+3 x_{3}\right) \\
-\left(x_{3}\right)
\end{array}\right\} \\
\text { subject to } & 1.5 x_{1}-x_{2}+1.6 x_{3} \leq 9 \\
& x_{1}+2 x_{2}+x_{3} \leq 10 \\
& x_{i} \geq 0 \quad i=1,2,3 .
\end{array}
$$

The true nadir point for this problem is $\mathbf{z}^{\mathrm{nad}}=(94.5$, $96.3636,0,0)^{T}$. In [13], a close point $(94.4998,95.8747$, $0,0)^{T}$ was found using multiple, bi-objective optimization runs. The estimation is different in its second objective value by about $0.5 \%$. In the following, we show the results of our hybrid nadir point estimation procedure.

In Step 1 of the procedure, we find the ideal and worst objectives values: $(0,0,-31,-5.625)^{T}$ and $(97.5,100,0,0)^{T}$, respectively. (These values are obtained by using the SQP routine of MATLAB. A linear solver could have been used instead.)

Thereafter, in Step 2, we apply the extremized-crowded NSGA-II procedure initializing the population around $x_{i} \in$ $[0,10]$ for all three variables. NSGA-II is terminated when the change in the $N D$ value in the past 50 generations is below the threshold of $\Delta=0.0001$. Fig. 15 shows the change in the

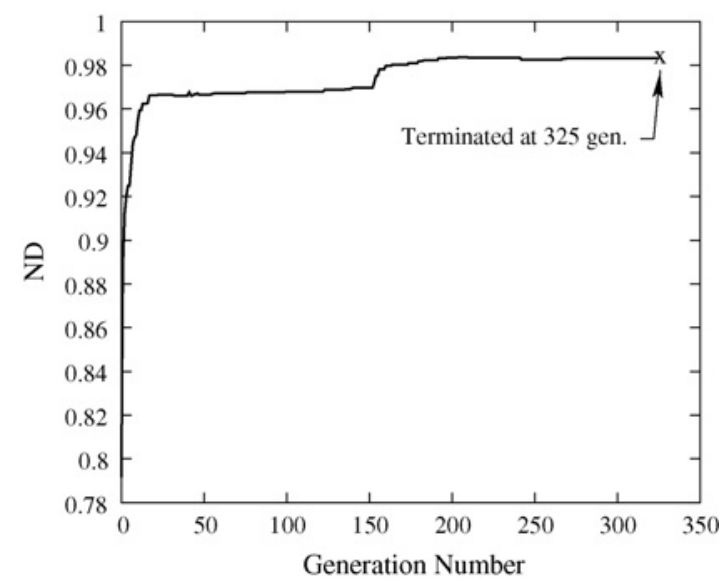

Fig. 15. Variation of $N D$ metric with generation for problem SW.

$N D$ value with the generation counter and indicates that the NSGA-II run was terminated at generation 325 . We obtain four different nondominated solutions, which are tabulated in Table IV. The minimum and maximum objective vectors are: $(0.0000,0.0000,-30.9920,-5.6249)^{T}$ and $(94.4810$, $96.3635,0.0000,0.0000)^{T}$, respectively. Notice that this maximum vector is close to the true nadir point mentioned above. We shall now investigate whether the proposed local search is able to improve this point to find the nadir point more accurately.

We observe that the first solution does not correspond to the worst value for any objective. Thus, in Step 3, we employ the bilevel local search procedure only for the other three solutions. The resulting solutions and corresponding $\mathbf{z}$ and $\mathbf{w}$ vectors are shown in the table. For solutions 2 and 3, we maximize objectives $f_{2}$ and $f_{1}$, respectively. Since solution 4 is worst with respect to both objectives $f_{3}$ and $f_{4}$, we maximize a normalized composite objective: $-\left[\left(f_{3}(\mathbf{x})-f_{3}^{\min }\right) /\left(f_{3}^{\max }-\right.\right.$ $\left.\left.f_{3}^{\min }\right)+\left(f_{4}(\mathbf{x})-f_{4}^{\min }\right) /\left(f_{4}^{\max }-f_{4}^{\min }\right)\right]$, where maximum and minimum objective values are those obtained by the modified NSGA-II in Step 2.

From the obtained local search solutions (the last column in the table), in Step 4, we estimate the nadir point as $(94.5000,96.3636,0,0)^{T}$, which is identical to the true nadir point for this problem. In this problem, the NSGA-II approach required 12640 solution evaluations out of an overall 13032 solution evaluations. Thus, the bilevel local search required only 392 solution evaluations (only about $3 \%$ of the overall effort). Thus, the use of an extremized-crowded NSGA-II allowed near critical points to be found by taking most of the computational effort and the use of the bilevel local search ensured finding the critical points by taking only a small 
TABLE IV

EXTREMIZED-Crowded NSGA-II AND LOCAL SEARCH METHOD ON PROBLEM SW

\begin{tabular}{|c|c|c|c|}
\hline & $\mathbf{x}_{\text {NSGA-II }}$ & Objective Vector, $\mathbf{f}_{\text {NSGA-II }}$ \\
\hline 1 & $(0.0001,0,5.6249)^{T}$ & $(42.1879,50.6249,-16.8752,-5.6249)^{T}$ & \\
2 & $(0.0001,3.1830,3.6336)^{T}$ & $(89.3219,96.3635,-26.8164,-3.6336)^{T}$ & \\
3 & $(3.9980,2.9998,0.0003)^{T}$ & $(94.4810,87.9854,-30.9920,-0.0003)^{T}$ & \\
4 & $(0,0,0)^{T}$ & $(0,0,0,0)^{T}$ & Extreme Point \\
\hline & $\mathbf{w}$ & $\mathbf{z}$ & $(89.3182,96.3636,-26.8182,-3.6364)^{T}$ \\
\hline 1 & \multicolumn{3}{|c|}{ Not worse in any objective, so not considered } \\
2 & $(1.0000,0.9844,0.7061,0.8232)^{T}$ & $(183.8020,192.7266,-26.8004,-3.6336)^{T}$ & $(89.000000)^{T}$ \\
3 & $(0.2958,0.2540,0.2006,0.2486)^{T}$ & $(188.9619,184.3489,-30.9920,5.6246)^{T}$ & $(94.5000,88.0000,-31.0000,0.0000,0,0,0)$ \\
4 & $(0.25,0.25,0.25,0.25)^{T}$ & $(0,0,0,0)^{T}$ & $(0,0,0,0)^{T}$ \\
\hline
\end{tabular}

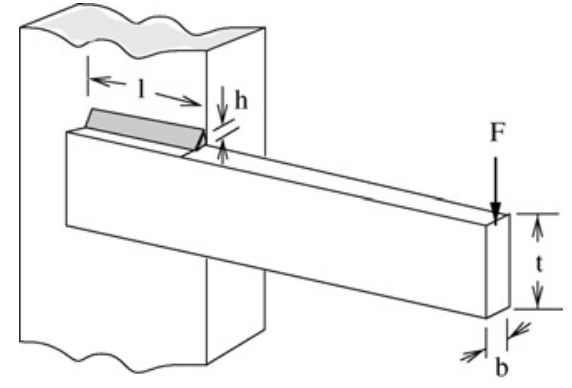

Fig. 16. Welded beam design problem.

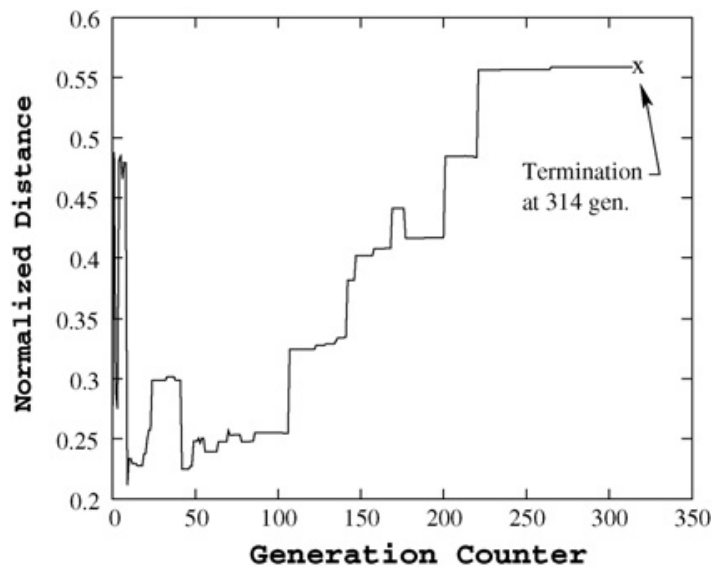

Fig. 17. Variation of $N D$ metric with generation for the welded beam design problem.

fraction of the overall computational effort, despite the bilevel nature of the optimization procedure.

\section{Welded Beam Design Optimization}

So far, we have applied the hybrid nadir point estimation procedure to numerical test problems. They have given us confidence about the usability of our procedure. Next, we consider an engineering design problem related to a welded beam having three objectives, for which the exact nadir point is not known. In this problem, we compare our proposed nadir point estimation procedure with the naive NSGA-II approach for the number of computations needed by each procedure and also to investigate whether an identical nadir point is estimated by each procedure.
This problem is well-studied [18], [32] having four design variables, $\mathbf{x}=(h, \ell, t, b)^{T}$ (dimensions specifying the welded beam). Minimizations of cost of fabrication, end deflection, and normal stress due to load $F=6000 \mathrm{lb}$ are of importance in this problem. There are four nonlinear constraints involving shear stress, normal stress, a physical property, and buckling limitation.

The mathematical description of the problem is given below

$$
\begin{array}{cl}
\text { minimize } & \left\{\begin{array}{l}
f_{1}(\mathbf{x})=1.10471 h^{2} \ell+0.04811 t b(14.0+\ell) \\
f_{2}(\mathbf{x})=\delta(\mathbf{x})=2.1952 / t^{3} b \\
f_{3}(\mathbf{x})=\sigma(\mathbf{x})=504000 / t^{2} b
\end{array}\right\} \\
\text { subject to } & g_{1}(\mathbf{x}) \equiv 13600-\tau(\mathbf{x}) \geq 0 \\
& g_{2}(\mathbf{x}) \equiv 30000-\sigma(\mathbf{x}) \geq 0 \\
& g_{3}(\mathbf{x}) \equiv b-h \geq 0 \\
& g_{4}(\mathbf{x}) \equiv P_{c}(\mathbf{x})-6000 \geq 0 \\
& 0.125 \leq \ell, t \leq 10 \\
& 0.125 \leq h, b \leq 5
\end{array}
$$

where the terms $\tau(\mathbf{x})$ and $P_{c}(\mathbf{x})$ are given as

$$
\begin{aligned}
\tau(\mathbf{x})= & {\left[\left(\tau^{\prime}(\mathbf{x})\right)^{2}+\left(\tau^{\prime \prime}(\mathbf{x})\right)^{2}+\ell \tau^{\prime}(\mathbf{x}) \tau^{\prime \prime}(\mathbf{x}) /\right.} \\
& \left.\sqrt{0.25\left(\ell^{2}+(h+t)^{2}\right)}\right]^{1 / 2} \\
P_{c}(\mathbf{x})= & 64,746.022(1-0.0282346 t) t b^{3}
\end{aligned}
$$

where

$$
\begin{aligned}
\tau^{\prime}(\mathbf{x}) & =\frac{6000}{\sqrt{2} h \ell} \\
\tau^{\prime \prime}(\mathbf{x}) & =\frac{6000(14+0.5 \ell) \sqrt{0.25\left(\ell^{2}+(h+t)^{2}\right)}}{2\left[0.707 h \ell\left(\ell^{2} / 12+0.25(h+t)^{2}\right)\right]} .
\end{aligned}
$$

In this problem, we have no knowledge on the ideal and worst objective values. Since these values will be required for computing the $N D$ metric value for terminating the extremized-crowded NSGA-II, we first find them here.

1) Step 1: Computing Ideal and Worst Objective Vectors: We minimize and maximize each of the three objectives to find the individual extreme points of the feasible objective space. For this purpose, we have used a single-objective realparameter genetic algorithm with the SBX recombination and the polynomial mutation operators [18], [28]. We use a different set of parameter values from that of our multiobjective NSGA-II studies: population size $=100$, maximum generations 
TABLE V

Minimum AND MaXimum ObJective VAlues of Three OBJectives

\begin{tabular}{|c|c|c|c|c|c|c|c|}
\hline & Cost & Deflection & Stress & $x_{1}$ & $x_{2}$ & $x_{3}$ & $x_{4}$ \\
\hline $\begin{array}{l}\text { Minimum } \\
\text { Min. after LS }\end{array}$ & $\begin{array}{l}2.3848 \\
2.3810\end{array}$ & & & $\begin{array}{l}0.2428 \\
0.2444\end{array}$ & $\begin{array}{l}6.2664 \\
6.2175\end{array}$ & $\begin{array}{l}8.2972 \\
8.2915\end{array}$ & $\begin{array}{l}0.2443 \\
0.2444\end{array}$ \\
\hline $\begin{array}{l}\text { Maximum } \\
\text { Max. after LS }\end{array}$ & $\begin{array}{l}333.9095 \\
333.9095\end{array}$ & & & $\begin{array}{l}5 \\
5\end{array}$ & $\begin{array}{l}10 \\
10\end{array}$ & $\begin{array}{l}10 \\
10\end{array}$ & $\begin{array}{l}5 \\
5\end{array}$ \\
\hline $\begin{array}{l}\text { Minimum } \\
\text { Min. after LS }\end{array}$ & & $\begin{array}{l}0.000439 \\
0.000439\end{array}$ & & $\begin{array}{l}\text { (*)4.4855 } \\
\text { (*)4.4855 }\end{array}$ & $\begin{array}{l}(*) 9.5683 \\
(*) 9.5683\end{array}$ & $\begin{array}{l}10 \\
10\end{array}$ & $\begin{array}{l}5 \\
5\end{array}$ \\
\hline $\begin{array}{l}\text { Maximum } \\
\text { Max. after LS }\end{array}$ & & $\begin{array}{l}0.0713 \\
0.0713\end{array}$ & & $\begin{array}{l}0.8071 \\
0.8071\end{array}$ & $\begin{array}{l}5.0508 \\
5.0508\end{array}$ & $\begin{array}{l}1.8330 \\
1.8330\end{array}$ & $\begin{array}{l}5 \\
5\end{array}$ \\
\hline $\begin{array}{l}\text { Minimum } \\
\text { Min. after LS }\end{array}$ & & & $\begin{array}{l}1008 \\
1008\end{array}$ & $\begin{array}{l}\text { (*)4.5959 } \\
\text { (*)4.5959 }\end{array}$ & $\begin{array}{l}\text { (*)9.9493 } \\
(*) 9.9493\end{array}$ & $\begin{array}{l}10 \\
10\end{array}$ & $\begin{array}{l}5 \\
5\end{array}$ \\
\hline $\begin{array}{l}\text { Maximum } \\
\text { Max. after LS }\end{array}$ & & & $\begin{array}{l}30000 \\
30000\end{array}$ & $\begin{array}{l}2.7294 \\
0.7301\end{array}$ & $\begin{array}{l}5.7934 \\
5.0376\end{array}$ & $\begin{array}{l}2.3255 \\
2.3308\end{array}$ & $\begin{array}{l}3.1066 \\
3.0925\end{array}$ \\
\hline
\end{tabular}

The values marked with a $\left(^{*}\right)$ for variables $x_{1}$ and $x_{2}$ can take other values without any change in the optimal objective value and without making the overall solution infeasible.

TABLE VI

\begin{tabular}{rrrr}
\hline & Cost & Deflection & Stress \\
\hline Ideal & 2.3810 & 0.000439 & 1008 \\
Worst & 333.9095 & 0.0713 & 30000 \\
\hline
\end{tabular}

$=500$, recombination probability $=0.9$, mutation probability $=$ 0.1 , distribution index for recombination $=2$, and distribution index for mutation $=20$. These values are usually followed in other single-objective real-parameter genetic algorithm (GA) studies [33], [34]. After a solution is obtained by a GA run, it is attempted to improve by a local search (LS) approachthe SQP procedure coded in MATLAB is applied with default parameter values to minimize individual objective functions in the feasible set. Table $\mathrm{V}$ shows the corresponding extreme objective values before and after the local search approaches.

Interestingly, the use of the local search improves the cost objective from 2.3848 to 2.3810 . As an outcome of the above single-objective optimization tasks, we obtain the ideal and worst objective values, as shown in Table VI.

2) Step 2: Applying Extremized-Crowded NSGA-II: First, we apply the extremized-crowded NSGA-II approach with an identical parameter setting as used above, except that for the SBX recombination $\eta_{c}=10$ is used, according to the recommendation in [18] for multiobjective optimization. The suggested termination criterion on the $N D$ metric is used with the above ideal and worst objective values. Fig. 17 shows the variation of the $N D$ metric with generation. It is interesting to note how the $N D$ metric, starting from a small value (meaning that the estimated nadir point is closer to the worst objective vector), reaches a stabilized value of 0.5587 . The NSGA-II procedure gets terminated at generation 314 .

Interestingly, only two nondominated extreme points are found by the extremized-crowded NSGA-II. They are shown in Table VII. From these two solutions, the estimated nadir point after Step 2 is $(36.4347,0.0169,28088.3266)^{T}$. In a threeobjective problem, the presence of only two extreme points signifies that two of the three objectives may be correlated to each other on the Pareto-optimal front. We shall discuss this aspect more later.

3) Step 3: Applying Local Search: The two solutions obtained are now attempted to be improved by the bilevel local search approach, one at a time. The minimum and maximum objective vectors obtained from the NSGA-II solutions (from Table VII) are as follows: $\mathbf{f}^{\min }=(2.8235,0.000439,1008)^{T}$ and $\mathbf{f}^{\max }=(36.4347,0.0169,28088.3266)^{T}$. Since the first solution corresponds to the worst of objective $f_{1}$, the upperlevel loop of the local search for solution 1 maximizes $f_{1}$. The resulting solution is shown in Table VII under the heading "After local search." A slightly better solution is obtained using the local search.

For solution 2 of Table VII, objectives $f_{2}$ and $f_{3}$ are both worst. Thus, we maximize a normalized quantity arising from both objectives: $\sum_{i=2}^{3}\left(f_{i}(\mathbf{x})-f_{i}^{\min }\right) /\left(f_{i}^{\max }-f_{i}^{\min }\right)$. The local search finds a nondominated solution which seems to be better in terms of the first two objectives but worse in the third objective. The weight vector obtained by the upper-level loop of the local search is $(0.2470,0.3333,0.4196)^{T}$ and the corresponding reference point is $(2.8235,0.0169,55168.65)^{T}$. An investigation will reveal that the local search utilized a reference point which has identical values for the first two objectives and a much worse $f_{3}$ value than the NSGA-II solution. Then, by using a weight vector which has more or less equal value for all three objectives, the upper loop is able to locate the critical point corresponding to the second and third objectives. Interestingly, this critical point corresponds to the minimum $f_{1}$ value which is exactly the same as that obtained by the minimization of the cost objective alone in Table V. It is clear that the extremized NSGA-II approach in Step 2 found a solution close to an extreme Pareto-optimal solution and the application of Step 3 helps to move this solution to the extreme Pareto-optimal solution.

Observing these two final solutions, in Step 4, we can now estimate the nadir point (cost, deflection, stress) for the welded beam design problem as

$$
\text { Nadir point: }(36.4209,0.0158,30000)^{T} \text {. }
$$

Note that this point is different from the worst objective vector of the entire feasible search space computed earlier. Out of a total of 31551 solution evaluations, the bilevel local search required only 51 solution evaluations, thereby demanding a tiny fraction of $0.16 \%$ of the overall computational effort. 
TABLE VII

Two Population Members Obtained Using the EXTREMized-Crowded NSGA-II APPRoACH

\begin{tabular}{|c|c|c|c|c|c|c|c|}
\hline Sol. No. & Cost & Deflection & Stress & $x_{1}$ & $x_{2}$ & $x_{3}$ & $x_{4}$ \\
\hline \multicolumn{8}{|c|}{ Extremized-crowded NSGA-II } \\
\hline 1. & 36.4347 & 0.000439 & 1008 & 1.5667 & 0.5389 & 10 & 5 \\
2. & 2.8235 & 0.0169 & 28088.3266 & 0.3401 & 4.6715 & 7.2396 & 0.3424 \\
\hline \multicolumn{8}{|c|}{ After Local Search } \\
\hline 1. & 36.4209 & 0.000439 & 1008 & 1.7345 & 0.4789 & 10 & 5 \\
2. & 2.3810 & 0.0158 & 30000 & 0.2444 & 6.2175 & 8.2915 & 0.2444 \\
\hline
\end{tabular}

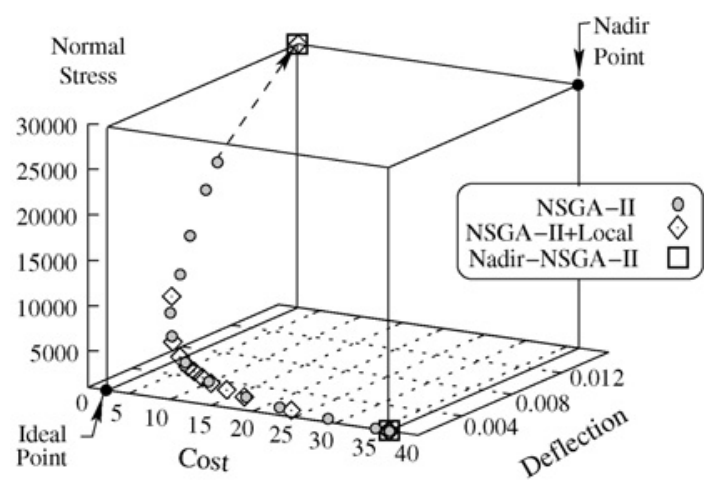

Fig. 18. Pareto-optimal front and estimation of the nadir point.

4) Comparison With the Naive NSGA-II Approach: We now apply the naive NSGA-II approach to the same problem to investigate whether an identical nadir point is obtained. In the naive approach, we first generate a set of Pareto-optimal points by a combination of the original NSGA-II and a local search approach. The range of the Pareto-optimal front, thus found, will provide us with information about the nadir point of the problem. We use an identical parameter setting as used in the extremized-crowded NSGA-II run. The local search approach used here is applied to NSGA-II solutions one at a time and is described in Chapter 9 (Section VI) of [18]. The resulting optimization problems are solved using the fmincon routine of MATLAB software. In Fig. 18, we show the NSGA-II solutions with circles and their improvements by the local search method with diamonds. The two nondominated extreme solutions obtained using our nadir point estimation procedure are marked using squares. Both approaches find an identical nadir point, thereby providing confidence to our proposed approach. However, the overall function evaluations needed to complete the naive NSGA-II and local searches for obtaining the distributed set of Pareto-optimal points were 102267 , compared to a total of 31551 function evaluations needed with our proposed nadir point estimation procedure. For a fourvariable, three-objective problem, a reduction of about $70 \%$ computations with our proposed approach to find an identical nadir point is a significant achievement.

It is also interesting to note that despite the use of three objectives, the Pareto-optimal front is 1-D in this problem. If the obtained front is projected on the deflection-stress $\left(f_{2}-f_{3}\right)$ plane, it can be seen that these two objectives are correlated to each other. Therefore, in addition to finding the nadir point, the number of extreme solutions $\mathbf{x}_{\mathrm{EA}}$ found by the extremized-crowded NSGA-II procedure may provide a rough idea about the dimensionality of the Pareto-optimal front-an added benefit which can be obtained by performing the nadir point estimation task before attempting to solve a problem for multiple Pareto-optimal solutions. A significant amount of research efforts is now being made in handling problems with many objective functions using evolutionary algorithms and in automatically identifying redundant objectives in a problem [21], [35], [36]. An analysis of critical points obtained by the proposed extremized-crowded NSGA-II procedure for identifying possible redundancy in objectives is worth pursuing further and remains as a viable approach in this direction.

\section{DISCUSSIONS AND EXTENSIONS}

In this paper, we have combined the flexibility in an EMO search with an ingenious local search procedure. By redirecting the focus of an EMO's diversity-preserving operator toward the extreme nondominated solutions, we have suggested an extremized-crowded NSGA-II procedure which is able to find representative points close to extreme points of the Paretooptimal front, not only to three or four-objective problems, but to as many as 20 -objective problems. By proposing a bilevel local search procedure of choosing an appropriate reference point near an obtained NSGA-II solution and a suitable weight vector for finding the critical point corresponding to the worst nondominated solutions obtained by the NSGA-II procedure, we have demonstrated the working of the hybrid procedure to a number of challenging test and practical optimization problems. Because we need a local optimization method for the bilevel problems, it is important to point out that a method most appropriate to the characteristics of the problem in question should always be favored.

To make NSGA-II's search more efficient, a mating restriction strategy can be added so that a better stability of multiple extreme solutions is maintained in the population. Restricting recombination among neighboring solutions in the objective space may also allow a focused search, thereby finding a better approximation of extreme solutions. For this purpose, the emphasis for extreme solutions can also be implemented on other EMO procedures, such as strength Pareto evolutionary algorithm-2 [37], Pareto envelope based selection algorithm [38], or others.

In the upper-level local search approach [problem (7)], the upper bound on the reference point $\mathbf{z}$ is chosen rather loosely. Since the task is to perform a local search, a tighter and more problem-informatic upper bound, such as a more relaxed bound on the worst objective value and a more restricted bound 
on the other objectives can be used for a computationally faster procedure. Similarly, the bounds on the weight vector can also be chosen with some problem information derived from the location of the particular NSGA-II solution vis-a-vis other solutions. In fact, based on the properties of achievement scalarizing functions, the inclusion of the weight vector $\mathbf{w}$ in the upper-level optimization is needed, but can be considered fixed (and not as a variable vector) as indicated in [39]. In that approach, by fixing the weight vector based on the location of the NSGA-II solution, the upper-level optimization may be used to find an optimal $\mathbf{z}$ corresponding to the extreme Pareto-optimal solution. This task typically requires less computational effort due to the reduction in decision variables on the upper-level optimization loop and remains an interesting future study to test further.

In another approach, the bilevel local search procedure suggested here can be integrated within the NSGA-II procedure as an additional operator. The local search can be applied to a few selected solutions of a NSGA-II population after every few generations. This on-line procedure will guarantee finding (locally) Pareto-optimal solutions whenever the local search is applied. A preliminary study [40] has shown some promising results in this direction. However, its computational advantage on more complex problems, if any, compared to the proposed hybrid approach of this paper will be an interesting future research worth pursuing.

\section{CONCLUSION}

We have proposed a hybrid methodology involving evolutionary and local search approaches to address an age-old yet open research issue of estimating the nadir point accurately in a multiobjective optimization problem. By definition, a nadir point is constructed from the worst objective values corresponding to the solutions of the Pareto-optimal front. It has been argued that the estimation of the nadir point is an important task in multiobjective optimization. Since the nadir point relates to the critical Pareto-optimal points, the estimation of a nadir point is also a difficult and challenging task. Since intermediate Pareto-optimal solutions are not important in this task, the suggested modified NSGA-II approaches have emphasized their search for finding the worst or extreme solutions corresponding to each objective. To enhance the convergence properties and make the approaches reliable, the modified NSGA-II approaches have been combined with a reference-point based bilevel local search approach. The upper-level search uses a combination of a reference point and a weight vector as a variable vector, which is then evaluated by using a lower-level search of solving the corresponding achievement scalarizing function. While the lower-level search is guaranteed to converge to a locally Pareto-optimal solution, the upper-level search drives the procedure to converge to the critical point of an objective function.

The extremized-crowded approach has been found to be capable of making a quicker estimate of the nadir point than a naive approach (of employing the original NSGA-II approach to first find a set of nondominated solutions and then construct the nadir point) on a number of benchmark problems having three to 20 objectives and on other problems including a difficult engineering design problem involving nonlinear objectives and constraints. Emphasizing solutions corresponding to the extreme objective values on a nondominated front has been found to be a better approach than emphasizing solutions having the worst objective values alone. Since the former approach maintains a diverse set of solutions near both best and worst objective values, thereby not allowing spurious dominated solutions to remain in the population, the result of the search is better and more reliable than that of the worstcrowded approach.

The computational effort to estimate the nadir point has been observed to be much smaller (more than an order of magnitude) for benchmark test problems having a large number of objectives than the naive NSGA-II approach. Moreover, since the extremized-crowded NSGA-II approach has been able to find solutions close to the critical points, the local search procedure has been found to take only a fraction of the overall computational effort. Thus, the bilevel nature of the proposed local search procedure does not seem to affect much the overall computational effort of the hybrid approach.

Despite the algorithmic challenge posed by the task of estimating the nadir point in a multiobjective optimization problem, in this paper, we have listed a number of reasons for which nadir objective vectors are useful in practice. They included normalizing objective functions, giving information about the ranges of objective functions within the Paretooptimal front to the decision maker, visualizing Pareto-optimal solutions, and enabling the decision maker to use different interactive methods. What is common to all these is that the nadir objective vector can be computed beforehand, without involving the decision maker. Thus, it is not a problem if several hundred function evaluations are needed in the extremizedcrowded NSGA-II in most problems. Approximating the nadir point can be an independent task to be executed before performing any decision analysis.

One of the reasons why it may be advisable to use some interactive method for identifying the most preferred solution instead of trying to approximate the whole set of Paretooptimal solutions is that for problems with several objectives, for example, the NSGA-II approach requires a huge number of evaluations to find a representative set. For such problems, the nadir point may be estimated quickly and reliably using the proposed hybrid NSGA-II-cum-local-search procedure. The extremized-crowded NSGA-II approach can be applied with a coarse termination requirement, so as to obtain near extreme nondominated solutions quickly. Then, the suggested local search approach can be employed to converge to the extreme Pareto-optimal solutions reliably and accurately. Thereafter, an interactive procedure (like NIMBUS [1], [4], [41], for example) (using both ideal and nadir points obtained) can be applied interactively with a decision-maker to find a desired Pareto-optimal solution as the most preferred solution. Alternatively, an evolutionary algorithm utilizing preference information such as [42] can be used. In this case, the nadir point together with the ideal point will inform the decisionmaker about the ranges of the objective and help the decision 
maker to concentrate on generating representations of desired parts of the Pareto front.

This paper is important in another aspect, as well. The proposed nadir point estimation procedure uses a hybridization of EMO and a local search based MCDM approach. The population aspect of EMO has been used to find near extreme nondominated solutions simultaneously and the referencepoint based local search methodology helped converge to true extreme Pareto-optimal solutions so that the nadir point can be estimated reliably and accurately. Such collaborative EMOMCDM studies may help develop efficient hybrid procedures which use best aspects of both contemporary fields of multiobjective optimization. Hopefully, this paper will motivate researchers to engage in more such collaborative studies for the benefit of either field and, above all, to the triumph of the field of multiobjective optimization.

\section{REFERENCES}

[1] K. Miettinen, Nonlinear Multiobjective Optimization. Boston, MA: Kluwer, 1999.

[2] K. Miettinen, M. M. Mäkelä, and K. Kaario, "Experiments with classification-based scalarizing functions in interactive multiobjective optimization," Eur. J. Oper. Res., vol. 175, no. 2, pp. 931-947, 2006.

[3] J. T. Buchanan, "A naive approach for solving MCDM problems: The GUESS method," J. Oper. Res. Soc., vol. 48, no. 2, pp. 202-206, 1997.

[4] K. Miettinen and M. M. Mäkelä, "Synchronous approach in interactive multiobjective optimization," Eur. J. Oper. Res., vol. 170, no. 3, pp. 909-922, 2006.

[5] K. Deb and S. Chaudhuri, "I-MODE: An interactive multiobjective optimization and decision-making using evolutionary methods," in Proc. 4th Int. Conf. Evol. Multi-Criteria Optimization (EMO), Berlin, Germany: Springer-Verlag, 2007, pp. 788-802.

[6] J. Branke, K. Deb, K. Miettinen, and R. Slowinski, Eds. Multiob jective Optimization: Interactive and Evolutionary Approaches. Berlin, Germany: Springer-Verlag, 2008.

[7] K. Miettinen, "Graphical illustration of Pareto optimal solutions," in Multi-Objective Programming and Goal Programming: Theory and Applications, T. Tanino, T. Tanaka, and M. Inuiguchi, Eds. Berlin, Germany: Springer-Verlag, 2003, pp. 197-202.

[8] R. Benayoun, J. de Montgolfier, J. Tergny, and O. Laritchev, "Linear programming with multiple objective functions: Step method (STEM)," Math. Programming, vol. 1, no. 3, pp. 366-375, 1971.

[9] H. Isermann and R. E. Steuer, "Computational experience concerning payoff tables and minimum criterion values over the efficient set," Eur. J. Oper. Res., vol. 33, no. 1, pp. 91-97, 1988.

[10] M. I. Dessouky, M. Ghiassi, and W. J. Davis, "Estimates of the minimum nondominated criterion values in multiple-criteria decision-making," Eng. Costs Product. Econ., vol. 10, no. 2, pp. 95-104, Jun. 1986.

[11] P. Korhonen, S. Salo, and R. Steuer, "A heuristic for estimating nadir criterion values in multiple objective linear programming," Oper. Res., vol. 45 , no. 5, pp. 751-757, 1997.

[12] M. Ehrgott and D. Tenfelde-Podehl, "Computation of ideal and nadir values and implications for their use in MCDM methods," Eur. J. Oper. Res., vol. 151, no. 1, pp. 119-139, Nov. 2003.

[13] M. Szczepanski and A. P. Wierzbicki, "Application of multiple criterion evolutionary algorithm to vector optimization, decision support and reference-point approaches," J. Telecommun. Inf. Technol., vol. 3, no. 3 , pp. $16-33,2003$

[14] H. P. Benson, "Existence of efficient solutions for vector maximization problems," J. Optimization Theory Applicat., vol. 26, no. 4, pp. 569-580, 1978.

[15] K. Deb, S. Chaudhuri, and K. Miettinen, "Toward estimating nadir objective vector using evolutionary approaches," in Proc. Genetic Evol. Comput. Conf. (GECCO), New York: The Association of Computing Machinery, 2006, pp. 643-650.

[16] K. Deb, S. Agrawal, A. Pratap, and T. Meyarivan, "A fast and elitist multiobjective genetic algorithm: NSGA-II," IEEE Trans. Evol. Comput., vol. 6, no. 2, pp. 182-197, Apr. 2002.

[17] A. P. Wierzbicki, "The use of reference objectives in multiobjective optimization," in Multiple Criteria Decision Making Theory and Appli- cations, G. Fandel and T. Gal, Eds. Berlin, Germany: Springer-Verlag, 1980, pp. 468-486.

[18] K. Deb, Multi-Objective Optimization Using Evolutionary Algorithms. Chichester, U.K.: Wiley, 2001.

[19] C. A. C. Coello, D. A. VanVeldhuizen, and G. Lamont, Evolutionary Algorithms for Solving Multi-Objective Problems, 2nd ed. Boston, MA: Kluwer, 2007.

[20] V. Khare, X. Yao, and K. Deb, "Performance scaling of multiobjective evolutionary algorithms," in Proc. 2nd Evol. Multi-Criterion Optimization (EMO) Conf., LNCS 2632. Berlin, Germany: Springer-Verlag, 2003, pp. 376-390.

[21] K. Deb and D. Saxena, "Searching for Pareto-optimal solutions through dimensionality reduction for certain large-dimensional multiobjective optimization problems," in Proc. World Congr. Comput. Intell. (WCCI), New York: IEEE Press, 2006, pp. 3352-3360.

[22] D. W. Corne and J. D. Knowles, "Techniques for highly multiobjective optimisation: Some nondominated points are better than others," in Proc. 9th Annu. Conf. Genetic Evol. Comput. (GECCO), New York: ACM, 2007, pp. 773-780.

[23] K. Deb and D. E. Goldberg, "An investigation of niche and species formation in genetic function optimization," in Proc. 3rd Int. Conf. Genetic Algorithms, San Francisco, CA: Morgan Kaufmann, 1989, pp. $42-50$.

[24] K. Deb, "Multiobjective genetic algorithms: Problem difficulties and construction of test problems," Evol. Comput., vol. 7, no. 3, pp. 205230, 1999.

[25] M. Laumanns, L. Thiele, K. Deb, and E. Zitzler, "Combining convergence and diversity in evolutionary multiobjective optimization," Evol. Comput., vol. 10, no. 3, pp. 263-282, 2002.

[26] K. Sindhya, K. Deb, and K. Miettinen, "A local search based evolutionary multiobjective optimization technique for fast and accurate convergence," in Proc. Parallel Problem Solving Nature (PPSN), Berlin, Germany: Springer-Verlag, 2008, pp. 815-824.

[27] M. Luque, K. Miettinen, P. Eskelinen, and F. Ruiz, "Incorporating preference information in interactive reference-point based methods for multiobjective optimization," Omega, vol. 37, no. 2, pp. 450-462, 2009.

[28] K. Deb and R. B. Agrawal, "Simulated binary crossover for continuous search space," Complex Syst., vol. 9, no. 2, pp. 115-148, 1995.

[29] K. Deb, L. Thiele, M. Laumanns, and E. Zitzler, "Scalable test problems for evolutionary multiobjective optimization," in Evolutionary Multiobjective Optimization: Theoretical Advances and Applications, A. Abraham, L. Jain, and R. Goldberg, Eds. London, U.K.: SpringerVerlag, 2005, pp. 105-145.

[30] K. Klamroth and K. Miettinen, "Integrating approximation and interactive decision making in multicriteria optimization," Oper. Res., vol. 56, no. 1, pp. 222-234, 2008.

[31] P. Eskelinen, K. Miettinen, K. Klamroth, and J. Hakanen, "Pareto navigator for interactive nonlinear multiobjective optimization," $O R$ Spectrum, vol. 32, no. 1, pp. 211-227, 2010.

[32] G. V. Reklaitis, A. Ravindran, and K. M. Ragsdell, Engineering Optimization Methods and Applications. New York: Wiley, 1983.

[33] K. Deb and M. Goyal, "A robust optimization procedure for mechanical component design based on genetic adaptive search," Trans. Am. Soc. Mech. Eng. J. Mech. Design, vol. 120, no. 2, pp. 162-164, 1998.

[34] K. Deb, "Mechanical component design using genetic algorithms," in Evolutionary Algorithms in Engineering Applications, D. Dasgupta and Z. Michalewicz, Eds. New York: Springer-Verlag, 1997, pp. 495-512.

[35] D. Brockhoff and E. Zitzler, "Dimensionality reduction in multiobjective optimization: The minimum objective subset problem," in Proc. Operations Res. 2006, Berlin, Germany: Springer, 2007, pp. 423-429.

[36] A. L. Jaimes, C. A. C. Coello, and D. Chakraborty, "Objective reduction using a feature selection technique," in Proc. Genetic Evol. Comput. Conf. (GECCO), New York: ACM, 2008, pp. 673-680.

[37] E. Zitzler, M. Laumanns, and L. Thiele, "SPEA2: Improving the strength Pareto evolutionary algorithm for multiobjective optimization," in Evolutionary Methods for Design, Optimization and Control With Applications to Industrial Problems, K. C. Giannakoglou, D. T. Tsahalis, J. Périaux, K. D. Papailiou, and T. Fogarty, Eds. Barcelona, Spain: CIMNE, 2001, pp. 95-100.

[38] D. W. Corne, J. D. Knowles, and M. Oates, "The Pareto envelopebased selection algorithm for multiobjective optimization," in Proc. 6th Int. Conf. Parallel Problem Solving Nature (PPSN), Berlin, Germany: Springer-Verlag, 2000, pp. 839-848.

[39] K. Deb and K. Miettinen, "Nadir point estimation using evolutionary approaches: Better accuracy and computational speed through focused search," in Multiple Criteria Decision Making for Sustainable Energy 
and Transportation Systems, M. Ehrgott, B. Naujoks, T. J. Stewart, and J. Wallenius, Eds. Berlin, Germany: Springer, 2009, pp. 339-354.

[40] K. Deb, K. Miettinen, and D. Sharma, "A hybrid integrated multiobjective optimization procedure for estimating nadir point," in Proc. 5th Int. Conf. Evol. Multi-Criterion Optimization (EMO), Berlin, Germany: Springer, 2009, pp. 569-583.

[41] K. Miettinen and M. M. Mäkelä, "Interactive bundle-based method for nondifferentiable multiobjective optimization: NIMBUS," Optimization, vol. 34, no. 3, pp. 231-246, 1995.

[42] L. Thiele, K. Miettinen, P. Korhonen, and J. Molina, "A preference-based interactive evolutionary algorithm for multiobjective optimization," Evol. Comput., vol. 17, no. 3, pp. 411-436, 2010.

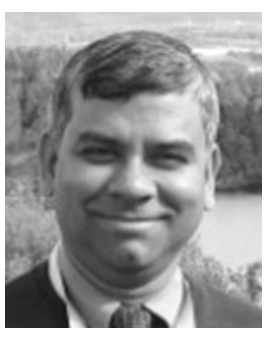

Kalyanmoy Deb received the Bachelor's degree from the Indian Institute of Technology (IIT) Kharagpur, Kharagpur, India, in 1985. Thereafter, he received the Master's and Doctoral degrees, both in engineering mechanics, from the University of Alabama, Tuscaloosa, in 1989 and 1991, respectively.

He was with Engineers India, Ltd., New Delhi during 1985-1987. After Ph.D. studies, he worked as a Visiting Research Assistant Professor with the General Engineering Department, University of Illinois at Urbana-Champaign, Urbana-Champaign. Since 1993, he has been teaching at IIT Kanpur. He is currently the Deva Raj Chair Professor with the Department of Mechanical Engineering, IIT Kanpur, Kanpur, India. He is also with the Aalto University School of Economics, Aalto, Finland. He has written two text books on optimization and more than 245 international journal and conference research papers. He is involved with editorial boards of 15 international journals of repute. More information about his research can be found at http://www.iitk.ac.in/kangal/deb.htm. His main research interests include computational optimization, modeling and design, and evolutionary algorithms.

Dr. Deb is the recipient of the prestigious Shanti Swarup Bhatnagar Prize in Engineering Sciences for the year 2005. He has also received the "Thomson Citation Laureate Award" from Thompson Scientific for having highest number of citations in computer science during the past ten years in India. $\mathrm{He}$ is a Fellow of the Indian National Academy of Engineering, the Indian National Academy of Sciences, and the International Society of Genetic and Evolutionary Computation. He also received the Fredrick Wilhelm Bessel Research Award from the Alexander von Humboldt Foundation in 2003.

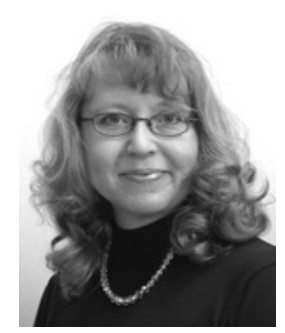

Kaisa Miettinen received the Master of Science degree in mathematics in 1988, the Licentiate of Philosophy degree in mathematical information technology in 1990, and the Doctor of Philosophy degree in mathematical information technology in 1994, all from the University of Jyväskylä, Jyväskylä, Finland.

Among others, she was with the International Institute for Applied Systems Analysis, Laxenburg, Austria in 1998, in different research and teaching positions funded by the Academy of Finland and the University of Jyväskylä, and as a Professor of financial mathematics with the Helsinki School of Economics, Helsinki, Finland, during 2004-2007. Currently, she is an Adjunct Professor with the Aalto University School of Economics and she is also a Professor of industrial optimization with the Department of Mathematical Information Technology, University of Jyväskylä, Jyväskylä, Finland, where she heads the Research Group on Industrial Optimization. The WWW-NIMBUS system, available at http://nimbus.it.jyu.fi at the University of Jyväskylä since 1995, was the first interactive multiobjective optimization software operating via the Internet. She has written the monograph Nonlinear Multiobjective Optimization (Kluwer/Springer, 1999) and over 50 peer-reviewed journal articles, as well as many peer-reviewed papers in collections and conference proceedings. Her research interests include multiobjective optimization (theory, methods, and software), multiple criteria decision making, nonlinear programming, evolutionary algorithms, hybrid approaches, as well as various applications of optimization.

Ms. Miettinen is the Co-Editor of the collection Multiobjective Optimization: Interactive and Evolutionary Approaches (Springer, 2008). She is the President-Elect of the International Society on Multiple Criteria Decision Making, the General Vice Chair and Chair-Elect of the Continuous Optimization Working Group of European Operational Research Societies, and a Member of the Steering Committee of Evolutionary Multicriterion Optimization. She has been the Co-Organizer of three Dagstuhl seminars devoted to multiobjective optimization, bringing together researchers from both multiple criteria decision making and evolutionary multiobjective optimization fields. She is the General Chair of the 21st International Conference on Multiple Criteria Decision Making to be held in June 2011 in Jyväskylä. She has received the Vaisala Award of the Finnish Academy of Science and Letters in the field of mathematics. Her homepage is http://www.mit.jyu.fi/miettine/engl.html.

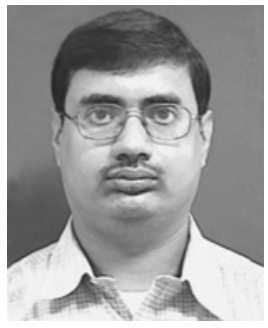

Shamik Chaudhuri received the Bachelor's degree in mechanical engineering from Jadavpur University, Kolkata, India, in 1999, the Master's degree in mechanical engineering from the Indian Institute of Technology (IIT) Kanpur, Kanpur, India, in 2002, and the Ph.D. degree in mechanical engineering from the IIT Kanpur, in 2006. During his Masters and Doctoral studies, he specialized in optimization, specifically in evolutionary algorithms.

Previously, he was with Larsen \& Toubro, Mumbai, India, during 1999-2000, and with TATA Motors, Mumbai, India, at their Engineering Research Center during 2002 2003. Currently, he is with the General Electric India Technology Center, Bangalore, India, where he works in the field of optimization and robust design. His main research interests include evolutionary algorithms, optimal system design, and meta-modeling. 